\title{
Glucagon-like peptide-I receptor agonists: a systematic review of comparative effectiveness research
}

This article was published in the following Dove Press journal: Diabetes, Metabolic Syndrome and Obesity:Targets and Therapy 4 April 2017

Number of times this article has been viewed

\author{
Philip A Levin' \\ Hiep Nguyen ${ }^{2}$ \\ Eric T Wittbrodt ${ }^{2}$ \\ Seoyoung $\mathrm{C} \mathrm{Kim}^{3}$
}

'Bay West Endocrinology Associates, Baltimore, MD, ${ }^{2} \mathrm{Health}$ Economics and Outcomes Research, AstraZeneca, Wilmington, DE, ${ }^{3}$ Division of Pharmacoepidemiology and Pharmacoeconomics, Department of Medicine, Brigham and Women's Hospital and Harvard Medical School, Boston, MA, USA
Correspondence: Seoyoung C Kim Division of Pharmacoepidemiology and Pharmacoeconomics, Department of Medicine, Brigham and Women's Hospital, I620 Tremont Street, Suite 3030, Boston, MA 02120, USA

$\mathrm{Tel}+\mathrm{I} 6172780930$

Fax +I 6172328602

Email skim62@partners.org
Background: Glucagon-like peptide-1 receptor agonists (GLP-1RAs) act by increasing insulin secretion, decreasing glucagon secretion, slowing gastric emptying, and increasing satiety.

Objective: Published evidence directly comparing GLP-1RAs with other approved treatments for type 2 diabetes (T2D) was systematically reviewed.

Methods: A literature search was performed using MEDLINE and Embase databases to identify papers comparing GLP-1RAs with other classes of glucose-lowering therapy in patients with T2D. Results: Of the 1303 papers identified, 57 met the prespecified criteria for a high-quality clinical trial or retrospective study. The efficacy and tolerability of approved GLP-1RAs (exenatide twice daily or once weekly, dulaglutide, liraglutide, lixisenatide, and albiglutide) were compared with insulin products (23 prospective studies + seven retrospective studies), dipeptidyl peptidase-4 inhibitors (11 prospective studies + three retrospective studies), sulfonylureas (nine prospective studies + one retrospective study), thiazolidinediones (five prospective studies), and metformin (two prospective studies). GLP-1RAs are effective as a second-line therapy in improving glycemic parameters in patients with T2D. Reductions in glycated hemoglobin from baseline with GLP-1RAs tended to be greater or similar compared with insulin therapy. GLP-1RAs were consistently more effective in reducing body weight than most oral glucose-lowering drugs and insulin and were associated with lower hypoglycemia risk versus insulin or sulfonylureas. GLP-1RAs improved cardiovascular risk factors, and preliminary data suggest they improve cardiovascular outcomes in patients with T2D compared with oral glucose-lowering drugs. However, results from ongoing studies are awaited to confirm these early findings.

Conclusion: This systematic review found that GLP-1RAs are an effective class of glucoselowering drugs for T2D.

Keywords: antidiabetic drugs, randomized controlled trials, retrospective, type 2 diabetes

\section{Introduction}

Diabetes mellitus is a chronic disease affecting a substantial proportion of the population. ${ }^{1}$ In adults (age 20-79 years), the 2015 prevalence of diabetes worldwide was estimated at $8.8 \%$, with type 2 diabetes (T2D) comprising $91 \%$ of cases. ${ }^{2}$ By 2030 , diabetes is expected to be the seventh leading cause of death. ${ }^{3}$

Several classes of glucose-lowering agents are currently available for the treatment of T2D, each with different mechanisms of action and therapeutic effects. Glucagonlike peptide-1 receptor agonists (GLP-1RAs) are a class of glucose-lowering drugs that act on the glucagon-like peptide-1 (GLP-1) receptor on pancreatic beta cells and increase insulin secretion, decrease glucagon secretion, slow gastric emptying, and increase satiety; clinical trials have shown that GLP-1RAs decrease body weight, 
postprandial glucose excursions, and some cardiovascular risk factors, without increasing the risk of hypoglycemia. ${ }^{4}$ Clinical trials have also studied the effects of GLP-1RA therapy on cardiovascular outcomes in patients with T2D. ${ }^{5,6}$

The purpose of this systematic review was to compare the efficacy of GLP-1RAs with other glucose-lowering therapies, using comparative data from clinical trials or observational cohort studies.

\section{Literature search strategy and filtering}

Embase and MEDLINE databases were searched on April 8, 2016, using the following search string: (glucagon like peptide 1 receptor agonist or "Glucagon-Like Peptide 1") OR (["Glucagon-like peptide-1" or "Glucagon-like peptide 1" or GLP1R or GLP-1 or GLP-1-R] AND [agonist or suppress* or block* or inhibit* or anti* or mimetic]) OR (lixisenatide or exenatide or liraglutide or albiglutide or dulaglutide) AND ([diabetes or diabetic or DM] and [“Type 2" or "Type-2" or "Type2" or "Type II" or "Type-II" or "Noninsulin-dependent" or "Noninsulin dependent" or "Non insulin-dependent" or "Non insulin dependent"]).

The search string was limited to keywords in the abstract or title. Articles indexed as animal studies, case reports, books, and conference/symposium presentations were excluded. The limits applied were English language and articles published between January 2005 and April 2016. Duplicates were removed, leaving 1303 articles. The articles identified were filtered manually and only those describing a head-to-head comparison between a GLP-1RA and another class of glucose-lowering therapy in $\geq 100$ patients, regardless of study design, follow-up duration, or medication doses, were included in the final total.

A total of 57 articles were included in the analysis (Figure 1); of these, 37 were randomized controlled trials (RCTs), seven were open-label extensions of RCTs, 11 were retrospective analyses, and two were prospective observational studies.

\section{Efficacy of GLP-I RAs versus other glucose-lowering therapies GLP-IRAs versus dipeptidyl peptidase-4 inhibitors (DPP-4is)}

Eleven prospective studies ${ }^{7-17}$ and three retrospective studies compared GLP-1RAs with DPP-4is. ${ }^{18-20}$

\section{Prospective studies}

Exenatide once weekly (QW) was associated with significantly greater $(P<0.001)$ reductions in glycated hemoglobin
(HbA1c) and fasting glucose (FG) compared with sitagliptin after 26 weeks in the Diabetes Therapy Utilization: Researching Changes in A1C, Weight and Other Factors Through Intervention with Exenatide Once Weekly (DURATION)-2 and DURATION-4 studies (Table 1). ${ }^{8,9}$ Although both exenatide QW and sitagliptin recipients lost weight, patients receiving exenatide $\mathrm{QW}$ had significantly greater weight loss from baseline. ${ }^{8,9}$ One study comparing exenatide twice daily (BID) with sitagliptin found that exenatide BID recipients had a reduction in FG similar to sitagliptin recipients, but a significantly greater reduction in weight. ${ }^{7}$

In the Assessment of Weekly Administration of LY2189265 in Diabetes (AWARD)-5 study, after 52 weeks of treatment, reductions from baseline in $\mathrm{HbAlc}, \mathrm{FG}$, and weight were significantly greater with dulaglutide than with sitagliptin (Table 1). ${ }^{10}$ These benefits were sustained over 104 weeks of treatment. ${ }^{11}$

Generally, liraglutide-treated patients had greater or similar reductions from baseline in $\mathrm{HbA1c}, \mathrm{FG}$, and weight compared with sitagliptin- or vildagliptin-treated patients (Table 1). ${ }^{12-14,16}$ In an open-label extension study, patients switching from sitagliptin to liraglutide had further reductions in these parameters. ${ }^{15}$

When administered for 24 weeks, lixisenatide produced reductions from baseline in $\mathrm{HbAlc}$ and $\mathrm{FG}$ that were similar to sitagliptin (Table 1 ) ${ }^{17}$ however, weight loss was significantly greater among lixisenatide versus sitagliptin recipients.

Hypoglycemia rates in patients receiving GLP-1RAs or DPP-4is were low, with only one instance of major/ severe hypoglycemia reported across all studies (in a patient receiving liraglutide $1.2 \mathrm{mg}$ ). ${ }^{13,14}$ In studies of exenatide QW, minor hypoglycemia rates ranged from $1 \%$ to $3.6 \%$ with exenatide QW, with the highest hypoglycemia rates for concomitant sulfonylurea use, and from $0 \%$ to $3.0 \%$ with DPP-4is. ${ }^{7-9}$ Patients receiving dulaglutide $1.5 \mathrm{mg}$ generally had numerically higher hypoglycemia rates than patients receiving dulaglutide $0.75 \mathrm{mg}$ or sitagliptin (10.2\% vs $5.3 \%$ and $4.8 \% ; 12.8 \%$ vs $8.6 \%$ and $8.6 \%$, respectively). ${ }^{10,11}$ Rates of hypoglycemia were similar for all liraglutide doses investigated versus DPP-4i comparators. ${ }^{12-14,16}$ Furthermore, the incidence of gastrointestinal adverse events (AEs; nausea, diarrhea, and vomiting) was higher ${ }^{7-11,13-15,17}$ or similar $^{16}$ in patients receiving GLP-1RAs versus DPP-4is; gastrointestinal AEs led to treatment withdrawal in a higher percentage of patients receiving GLP-1RA therapy in these studies. ${ }^{8,10,11}$ Some studies reported that gastrointestinal AEs peaked on treatment initiation in the GLP-1RA group and then stabilized over the study period. ${ }^{10,11,13}$ 


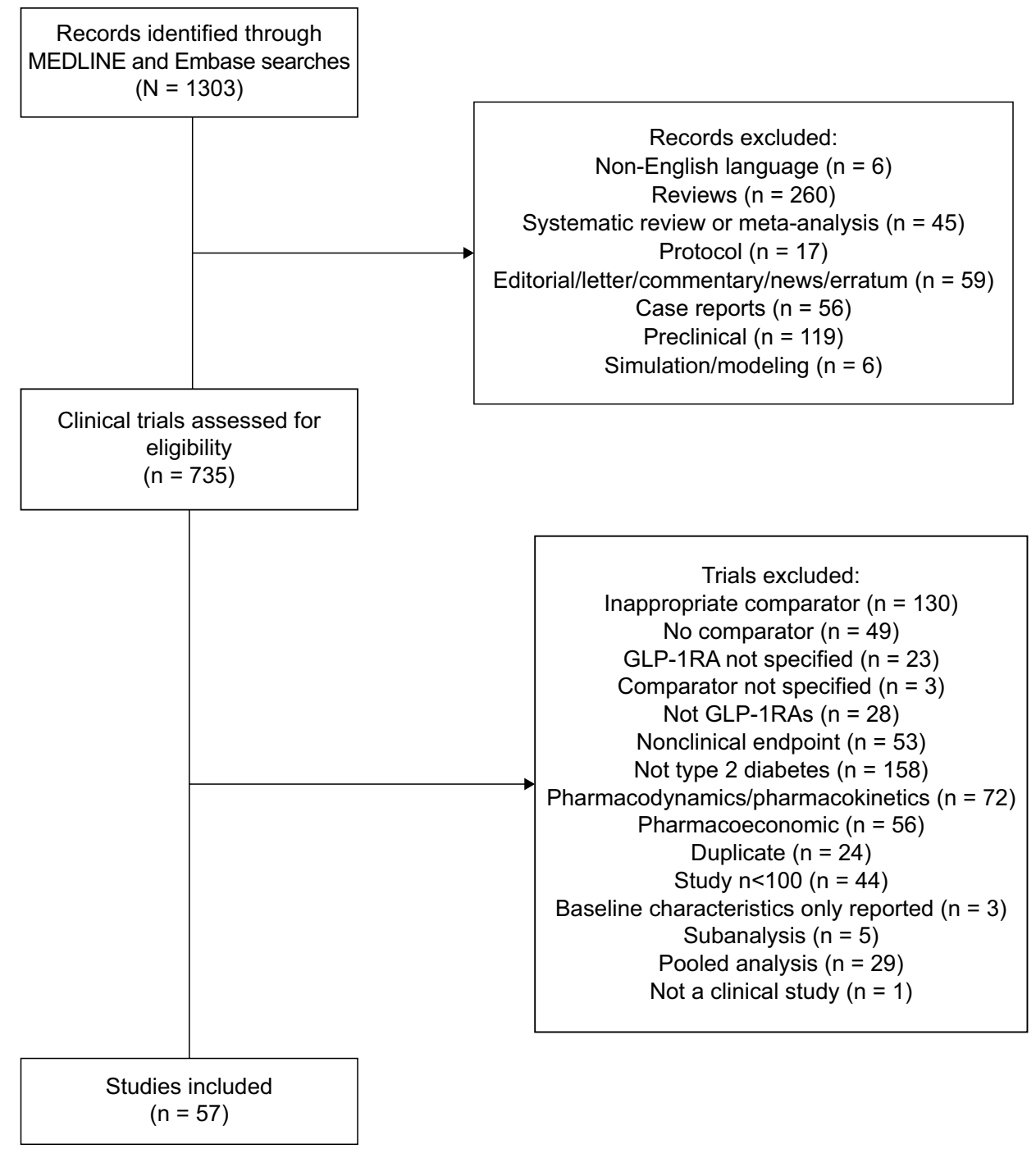

Figure I PRISMA flow diagram.

Abbreviation: GLP-IRA, glucagon-like peptide-I receptor agonist.

\section{Retrospective studies}

Retrospective studies had similar results to the prospective studies (Table 2), with GLP-1RAs demonstrating greater or similar reductions in $\mathrm{HbAlc}$, FG, and weight versus DPP-4is..$^{18-20}$ Generally, hypoglycemia rates were not reported, although in one study the rate was similar between GLP-1RAs and DPP-4is. ${ }^{19}$ Nausea/vomiting was more frequent with exenatide compared with sitagliptin (incidence rate, 0.39 vs 0.032 ) in one study. ${ }^{19}$

\section{GLP-IRAs versus metformin}

Two prospective studies comparing GLP-1RAs and metformin were identified. ${ }^{9,21}$

\section{Prospective studies}

Both studies (RCTs) reported reductions from baseline in HbAlc, FG, and weight (Table 1). ${ }^{9,21}$ In DURATION-4, exenatide $\mathrm{QW}$ and metformin reduced $\mathrm{HbA} 1 \mathrm{c}$ from baseline by $\sim 1.5 \%$, with no significant difference between groups at 26 weeks. In AWARD-3, dulaglutide $0.75 \mathrm{mg}$ and $1.5 \mathrm{mg}$ QW reduced $\mathrm{HbA} 1 \mathrm{c}$ significantly more than metformin $(P<$ $0.05)$ after 26 weeks. After 52 weeks, significant differences were observed in the reduction from baseline in $\mathrm{HbA} 1 \mathrm{c}$ between dulaglutide $1.5 \mathrm{mg}$ and metformin, but not between dulaglutide $0.75 \mathrm{mg}$ and metformin.

Two RCTs investigated the effects of exenatide QW and dulaglutide on FG and weight, with no significant differences between these drugs in the reductions of these endpoints at 26 weeks. ${ }^{9,21}$ Treatment with dulaglutide at both dosages resulted in similar reductions in FG versus metformin at 26 weeks; at 52 weeks, recipients of dulaglutide $1.5 \mathrm{mg}$ had a significantly greater reduction in $\mathrm{FG}$ than metformin recipients $(P<0.05$; Table 1). The magnitude of weight loss achieved in 
Table I Study details and efficacy results of comparative trials of GLP-IRAs and oral glucose-lowering therapies

\begin{tabular}{|c|c|c|c|c|c|c|}
\hline \multirow{2}{*}{$\begin{array}{l}\text { Publications } \\
\text { (acronym/ } \\
\text { ClinicalTrials.gov } \\
\text { record) }\end{array}$} & \multirow[t]{2}{*}{$\begin{array}{l}\text { Study design } \\
\text { (study duration) }\end{array}$} & \multirow[t]{2}{*}{$\begin{array}{l}\text { Treatment (patient } \\
\text { number) }\end{array}$} & \multirow[t]{2}{*}{$\begin{array}{l}\text { Background } \\
\text { therapy }\end{array}$} & \multicolumn{3}{|c|}{$\begin{array}{l}\text { Change from baseline at study end, GLP-IRAs versus } \\
\text { comparator }\end{array}$} \\
\hline & & & & HbAlc (\%) & FG (mmol/L) & BW (kg) \\
\hline \multicolumn{7}{|c|}{ Dipeptidyl peptidase-4 inhibitors } \\
\hline Berg et $\mathrm{al}^{7}$ & $\begin{array}{l}C O, D B, D D, R \\
\text { in pts with T2D } \\
(2 \times 4 \text { weeks })\end{array}$ & $\begin{array}{l}\text { ExBID I0 } \mu \mathrm{g} / \mathrm{SITA} 100 \mathrm{mg} \\
\text { QD (4I) } \\
\text { SITA I00 mg QD/ExBID } \\
10 \mu \mathrm{g}(42)\end{array}$ & $\begin{array}{l}\operatorname{MET}(n=82) \\
\operatorname{TZD}(n=1)\end{array}$ & NR & $\begin{array}{l}\text { ExBID: }-1.6 \\
\text { SITA: }-1.6\end{array}$ & $\begin{array}{l}\text { ExBID: }-1.4^{*} \\
\text { SITA: }-0.9\end{array}$ \\
\hline $\begin{array}{l}\text { Bergenstal et } \mathrm{al}^{8} \\
\text { (DURATION-2; } \\
\text { NCT00637273) }\end{array}$ & $\begin{array}{l}\mathrm{DB}, \mathrm{DD}, \mathrm{MC}, \mathrm{PG}, \\
\mathrm{R} \text { in pts with T2D } \\
(26 \text { weeks) }\end{array}$ & $\begin{array}{l}\text { ExQW } 2 \text { mg (I60) } \\
\text { SITA } 100 \text { mg QD (I66) } \\
\text { PIO } 45 \text { mg QD (165) }\end{array}$ & MET & $\begin{array}{l}\text { ExQW: }-1.5^{* * *} \\
\text { SITA: }-0.9\end{array}$ & $\begin{array}{l}\text { ExQW: }-1.8^{* *} \\
\text { SITA: }-0.9\end{array}$ & $\begin{array}{l}\text { ExQW: }-2.3^{* * *} \\
\text { SITA: }-0.8\end{array}$ \\
\hline $\begin{array}{l}\text { Russell-Jones et al }{ }^{9} \\
\text { (DURATION-4; } \\
\text { NCT00676338) }\end{array}$ & $\begin{array}{l}\mathrm{DB}, \mathrm{MC}, \mathrm{PG}, \mathrm{R} \text { in } \\
\text { treatment-naive } \\
\text { pts with T2D } \\
\text { (26 weeks) }\end{array}$ & $\begin{array}{l}\text { ExQW } 2 \text { mg (248) } \\
\text { MET } 2000 \text { mg/day (246) } \\
\text { PIO } 45 \text { mg QD (I63) } \\
\text { SITA } 100 \text { mg QD (I63) }\end{array}$ & None & $\begin{array}{l}\text { ExQW: }-1.5^{* * *} \\
\text { SITA: }-1.2\end{array}$ & $\begin{array}{l}\text { ExQW: }-2.3^{* * *} \\
\text { SITA: }-1 . I\end{array}$ & $\begin{array}{l}\text { ExQW: }-2.0^{* * * *} \\
\text { SITA: }-0.8\end{array}$ \\
\hline $\begin{array}{l}\text { Nauck et al }{ }^{10} \\
\text { (AWARD-5; } \\
\text { NCT00734474) }\end{array}$ & $\begin{array}{l}\mathrm{DB}, \mathrm{MC}, \mathrm{PG}, \mathrm{R} \\
\text { in pts with T2D } \\
\text { (52 weeks) }\end{array}$ & $\begin{array}{l}\text { DULA } 0.75 \mathrm{mg} \text { QW (302) } \\
\text { DULA I.5 mg QW (304) } \\
\text { SITA I00 mg QD (3I5) } \\
\text { PBO (I77) }\end{array}$ & MET & $\begin{array}{l}\text { DULA 0.75: }-0.9 * * * \\
\text { DULA I.5: }-1.1 * * * \\
\text { SITA: }-0.4\end{array}$ & $\begin{array}{l}\text { DULA } 0.75:-1.6^{* * *} \\
\text { DULA I.5: }-2.4^{* * * *} \\
\text { SITA: }-0.9\end{array}$ & $\begin{array}{l}\text { DULA } 0.75:-2.6 * * * \\
\text { DULA I.5: }-3.0^{* * *} \\
\text { SITA: }-1.5\end{array}$ \\
\hline $\begin{array}{l}\text { Weinstock et al" } \\
\text { (AWARD-5; } \\
\text { NCT00734474) }\end{array}$ & $\begin{array}{l}\mathrm{DB}, \mathrm{MC}, \mathrm{PG}, \mathrm{R} \\
\text { in pts with T2D } \\
\text { (104 weeks) }\end{array}$ & $\begin{array}{l}\text { DULA } 0.75 \mathrm{mg} \text { QW (302) } \\
\text { DULA I.5 mg QW (304) } \\
\text { SITA } 100 \mathrm{mg} \text { QD (3I5) } \\
\text { PBO (I77) }\end{array}$ & MET & $\begin{array}{l}\text { DULA } 0.75:-0.7 * * * \\
\text { DULA I.5: }-1.0 * * * \\
\text { SITA: }-0.3\end{array}$ & $\begin{array}{l}\text { DULA 0.75: } \\
-1.4^{* * *} \\
\text { DULA I.5: }-2.0^{* * *} \\
\text { SITA: }-0.5\end{array}$ & $\begin{array}{l}\text { DULA } 0.75:-2.4 \\
\text { DULA I.5: }-2.9 * * * \\
\text { SITA: }-1.8\end{array}$ \\
\hline $\begin{array}{l}\text { Charbonnel et al } \\
\text { (NCT0I2964I2) }\end{array}$ & $\begin{array}{l}\text { OL, MC, PG, R } \\
\text { in pts with T2D } \\
\text { (26 weeks) }\end{array}$ & $\begin{array}{l}\text { LIRA I. } 2 \mathrm{mg} / \text { day }^{\mathrm{a}}(253) \\
\text { SITA } 100 \mathrm{mg} \text { OD }^{\mathrm{b}}(269)\end{array}$ & MET & $\begin{array}{l}\text { LIRA: }-1.4 \\
\text { SITA: }-1.3\end{array}$ & $\begin{array}{l}\text { LIRA: }-2.2 \\
\text { SITA: }-1.9\end{array}$ & $\begin{array}{l}\text { LIRA: }-2.8 \\
\text { SITA: }-0.4\end{array}$ \\
\hline $\begin{array}{l}\text { Pratley et } \mathrm{al}^{13} \\
(\mathrm{NCT} 00700817)\end{array}$ & $\begin{array}{l}\text { OL, MC, PG, R } \\
\text { in pts with T2D } \\
\text { (52 weeks) }\end{array}$ & $\begin{array}{l}\text { LIRA } 1.2 \mathrm{mg} / \text { day }(225) \\
\text { LIRA } 1.8 \mathrm{mg} / \text { day }(22 \mathrm{I}) \\
\text { SITA } 100 \mathrm{mg} \text { QD }(2 \mathrm{I} 9)\end{array}$ & MET & $\begin{array}{l}\text { LIRA I.2: }-1.3^{* * *} \\
\text { LIRA I.8: }-1.5^{* * *} \\
\text { SITA: }-0.9\end{array}$ & $\begin{array}{l}\text { LIRA I.2: }-1.7^{* * * *} \\
\text { LIRA I.8: }-2.0^{* * *} \\
\text { SITA: }-0.6\end{array}$ & $\begin{array}{l}\text { LIRA I.2: }-2.8^{* * * *} \\
\text { LIRA I.8: }-3.7^{* * * *} \\
\text { SITA: }-1.2\end{array}$ \\
\hline $\begin{array}{l}\text { Pratley et al }{ }^{15} \\
(\text { NCT007008I7) }\end{array}$ & $\begin{array}{l}\text { OL extension } \\
\text { of pts with T2D } \\
\text { completing the } \\
\text { I860-LIRA- } \\
\text { DPP-4 core study } \\
\text { (26 weeks) }\end{array}$ & $\begin{array}{l}\text { SITA } 100 \mathrm{mg} \text { to LIRA } \\
1.2 \mathrm{mg}(67) \\
\text { SITA } 100 \mathrm{mg} \text { to LIRA } \\
1.8 \mathrm{mg}(68)\end{array}$ & MET & $\begin{array}{l}\text { LIRA I. } 2:-0.2 \dagger \\
\text { LIRA I.8: }-0.5 \dagger \dagger\end{array}$ & $\begin{array}{l}\text { LIRA I.2: }-0.8 \dagger \\
\text { LIRA I.8: }-1.4 \dagger \dagger\end{array}$ & $\begin{array}{l}\text { LIRA I.2: }-1.6+\dagger \\
\text { LIRA I.8: }-2.5+\dagger\end{array}$ \\
\hline Takeshita et al ${ }^{16}$ & $\begin{array}{l}\text { OL, PG, } R \text { in } \\
\text { Japanese pts with } \\
\text { T2D not adequately } \\
\text { controlled by } \\
\text { SITA-based } \\
\text { therapy ( } 12 \text { weeks) }\end{array}$ & $\begin{array}{l}\text { LIRA } 0.9 \text { mg QD (54) } \\
\text { VILD } 50 \text { mg BID (58) }\end{array}$ & None & $\begin{array}{l}\text { LIRA: }-0.7^{*} \\
\text { VILD: }-0.4\end{array}$ & $\begin{array}{l}\text { LIRA: }-1.0 \\
\text { VILD: }-0.8\end{array}$ & $\begin{array}{l}\text { LIRA: }-1.6^{* * *} \\
\text { VILD: }+0.1\end{array}$ \\
\hline $\begin{array}{l}\text { Van Gaal et al }{ }^{17} \\
(\text { NCT00976937) }\end{array}$ & $\begin{array}{l}\mathrm{DB}, \mathrm{DD}, \mathrm{MC}, \mathrm{PG}, \\
\mathrm{R} \text { in young, obese } \\
\text { pts with T2D } \\
\text { ( } 24 \text { weeks) }\end{array}$ & $\begin{array}{l}\text { LIXI } 20 \mu g \text { QD (I58) } \\
\text { SITA } 100 \text { mg QD (16I) }\end{array}$ & MET & $\begin{array}{l}\text { LIXI: }-0.7 \\
\text { SITA: }-0.7\end{array}$ & $\begin{array}{l}\text { LIXI: }-0.5 \\
\text { SITA: }-0.7\end{array}$ & $\begin{array}{l}\text { LIXI: }-2.5^{* * *} \\
\text { SITA: }-1.2\end{array}$ \\
\hline Metformin & & & & & & \\
\hline $\begin{array}{l}\text { Russell-Jones et al } \\
\text { (DURATION-4; } \\
\text { NCT00676338) }\end{array}$ & $\begin{array}{l}\mathrm{DB}, \mathrm{MC}, \mathrm{PG}, \mathrm{R} \text { in } \\
\text { treatment-naive } \\
\text { pts with T2D } \\
\text { (26 weeks) }\end{array}$ & $\begin{array}{l}\text { ExQW } 2 \text { mg (248) } \\
\text { MET } 2000 \text { mg/day (246) } \\
\text { PIO } 45 \text { mg QD (I63) } \\
\text { SITA } 100 \text { mg QD (I63) }\end{array}$ & None & $\begin{array}{l}\text { ExQW: }-1.5 \\
\text { MET: }-I .5\end{array}$ & $\begin{array}{l}\text { ExQW: }-2.3 \\
\text { MET: }-2.0\end{array}$ & $\begin{array}{l}\text { ExQW: }-2.0 \\
\text { MET: }-2.0\end{array}$ \\
\hline $\begin{array}{l}\text { Umpierrez et } \mathrm{al}^{21} \\
\text { (AWARD-3; } \\
\text { NCT0I I 26580) }\end{array}$ & $\begin{array}{l}\mathrm{DB}, \mathrm{DD}, \mathrm{MC}, \mathrm{PG}, \\
\mathrm{R} \text { in pts with T2D } \\
\left(52 \text { weeks }^{\mathrm{c}}\right)\end{array}$ & $\begin{array}{l}\text { DULA } 0.75 \mathrm{mg} \text { QW (270) } \\
\text { DULA I.5 mg QW (269) } \\
\text { MET } \geq 1500 \mathrm{mg} / \text { day }(268)\end{array}$ & None & $\begin{array}{l}\text { At } 26 \text { weeks } \\
\text { DULA } 0.75:-0.7^{*} \\
\text { DULA I.5: }-0.8^{* *} \\
\text { MET: }-0.6 \\
\text { At } 52 \text { weeks } \\
\text { DULA } 0.75:-0.6 \\
\text { DULA I.5: }-0.7^{*} \\
\text { MET: }-0.5\end{array}$ & $\begin{array}{l}\text { At } 26 \text { weeks } \\
\text { DULA } 0.75:-1.4 \\
\text { DULA I.5: }-1.6 \\
\text { MET: }-1.3 \\
\text { At } 52 \text { weeks } \\
\text { DULA } 0.75:-1.0 \\
\text { DULA } 1.5:-1.6^{*} \\
\text { MET: }-1.2\end{array}$ & $\begin{array}{l}\text { At } 26 \text { weeks } \\
\text { DULA } 0.75:-1.4^{* *} \\
\text { DULA I.5: }-2.3 \\
\text { MET: }-2.2 \\
\text { At } 52 \text { weeks } \\
\text { DULA } 0.75:-1 . I^{* * *} \\
\text { DULA I.5: }-1.9 \\
\text { MET: }-2.2\end{array}$ \\
\hline
\end{tabular}


Table I (Continued)

\begin{tabular}{|c|c|c|c|c|c|c|}
\hline \multirow{2}{*}{$\begin{array}{l}\text { Publications } \\
\text { (acronym/ } \\
\text { ClinicalTrials.gov } \\
\text { record) }\end{array}$} & \multirow[t]{2}{*}{$\begin{array}{l}\text { Study design } \\
\text { (study duration) }\end{array}$} & \multirow[t]{2}{*}{$\begin{array}{l}\text { Treatment (patient } \\
\text { number) }\end{array}$} & \multirow[t]{2}{*}{$\begin{array}{l}\text { Background } \\
\text { therapy }\end{array}$} & \multicolumn{3}{|c|}{$\begin{array}{l}\text { Change from baseline at study end, GLP-I RAs versus } \\
\text { comparator }\end{array}$} \\
\hline & & & & HbAlc (\%) & FG (mmol/L) & BW (kg) \\
\hline \multicolumn{7}{|l|}{ Sulfonylureas } \\
\hline Derosa et $\mathrm{a}^{122}$ & $\begin{array}{l}\text { MC, R, SB in } \\
\text { overweight pts } \\
\text { with T2D poorly } \\
\text { controlled on MET } \\
\text { (1 } 2 \text { months) }\end{array}$ & $\begin{array}{l}\text { ExBID } 10 \mu g^{d}(63) \\
\text { GLYB } 5 \text { mg } \text { TID }^{d}(65)\end{array}$ & MET & $\begin{array}{l}\text { ExBID: }-1.5 \\
\text { GLYB: }-1.8\end{array}$ & $\begin{array}{l}\text { ExBID: }-1.5 \\
\text { GLYB: }-1.8\end{array}$ & $\begin{array}{l}\text { ExBID: }-8.0^{* * *} \\
\text { GLYB: }+4.3\end{array}$ \\
\hline Derosa et $\mathrm{a}^{23}$ & $\begin{array}{l}\text { MC, R, SB in } \\
\text { overweight pts } \\
\text { with T2D poorly } \\
\text { controlled on MET } \\
\text { (1 } 2 \text { months) }\end{array}$ & $\begin{array}{l}\text { ExBID I0 } \mu g^{d}(57) \\
\text { GLIM } 2 \text { mg } \text { TID }^{d}(54)\end{array}$ & MET & $\begin{array}{l}\text { ExBID: }-1.2 \\
\text { GLIM: }-1.4\end{array}$ & $\begin{array}{l}\text { ExBID: }-1.5 \\
\text { GLIM: }-1.6\end{array}$ & $\begin{array}{l}\text { ExBID: }-5 . I \\
\text { GLIM: }-0.9\end{array}$ \\
\hline $\begin{array}{l}\text { Gallwitz et a }{ }^{24} \\
\text { (EUREXA; } \\
\text { NCT00359762) }\end{array}$ & $\begin{array}{l}\text { OL, } R, M C \text { in } \\
\text { overweight pts } \\
\text { with T2D poorly } \\
\text { controlled on MET } \\
\text { ( } \sim 3 \text { years })\end{array}$ & $\begin{array}{l}\text { ExBID I0 } \mu g^{d}(490) \\
\text { GLIM I mg TID }(487)\end{array}$ & MET & $\begin{array}{l}\text { ExBID: }-0.4^{* *} \\
\text { GLIM: }-0.2\end{array}$ & $\begin{array}{l}\text { ExBID: }-0.9 * \\
\text { GLIM: }-0.4\end{array}$ & $\begin{array}{l}\text { ExBID: }-3.3^{* * *} \\
\text { GLIM: }+1.2\end{array}$ \\
\hline $\begin{array}{l}\text { Nauck et } \mathrm{a}^{32} \\
\text { (LEAD-2; } \\
\text { NCT003 I846I) }\end{array}$ & $\begin{array}{l}\mathrm{DB}, \mathrm{DD}, \mathrm{MC}, \mathrm{PG}, \\
\mathrm{R} \text { in pts with T2D } \\
(26 \text { weeks })\end{array}$ & $\begin{array}{l}\text { LIRA } 0.6 \mathrm{mg} \text { QD (242) } \\
\text { LIRA I .2 mg QD (240) } \\
\text { LIRA I .8 mg QD (242) } \\
\text { GLIM } 4 \mathrm{mg} \text { QD (242) } \\
\text { PBO (I I I) }\end{array}$ & MET & $\begin{array}{l}\text { LIRA 0.6: }-0.7 \\
\text { LIRA I.2: }-1.0 \\
\text { LIRA I.8: }-1.0 \\
\text { GLIM 4: }-1.0\end{array}$ & $\begin{array}{l}\text { LIRA 0.6: }-I .1 \\
\text { LIRA I.2: }-1.6 \\
\text { LIRA I.8: }-I .7 \\
\text { GLIM 4: }-1.3\end{array}$ & $\begin{array}{l}\text { LIRA 0.6: }-1.8^{* * *} \\
\text { LIRA 1.2: }-2.6^{* * *} \\
\text { LIRA I.8: }-2.8^{* * *} \\
\text { GLIM 4: }+1.0\end{array}$ \\
\hline $\begin{array}{l}\text { Nauck et } \mathrm{a}^{28} \\
\text { (LEAD-2; } \\
\text { NCT003 I846I) }\end{array}$ & $\begin{array}{l}\text { OL extension } \\
\text { of pts with T2D } \\
\text { completing the } \\
\text { LEAD-2 core study } \\
\text { (18 months) }\end{array}$ & $\begin{array}{l}\text { LIRA } 0.6 \mathrm{mg} \text { QD (I84) } \\
\text { LIRA I.2 mg QD (I78) } \\
\text { LIRA I.8 mg QD (I74) } \\
\text { GLIM } 4 \text { mg QD (I83) } \\
\text { PBO (6I) }\end{array}$ & MET & $\begin{array}{l}\text { LIRA 0.6: }-0.4 \\
\text { LIRA I.2: }-0.6 \\
\text { LIRA I.8: }-0.6 \\
\text { GLIM 4: }-0.5\end{array}$ & $\begin{array}{l}\text { LIRA 0.6: }-0.8 \\
\text { LIRA I.2: }-1.2^{*} \\
\text { LIRA I.8: }-1.2 \\
\text { GLIM 4: }-0.6\end{array}$ & $\begin{array}{l}\text { LIRA 0.6: }-2.1^{* * *} \\
\text { LIRA I.2: }-3.0^{* * * *} \\
\text { LIRA I.8: }-2.9^{* * *} \\
\text { GLIM 4: }+0.7\end{array}$ \\
\hline $\begin{array}{l}\text { Garber et } \mathrm{al}^{25} \\
\text { (LEAD-3 Mono; } \\
\text { NCT00294723) }\end{array}$ & $\begin{array}{l}\mathrm{DB}, \mathrm{DD}, \mathrm{MC}, \mathrm{PG}, \\
\mathrm{R} \text { in pts with early } \\
\text { T2D (52 weeks) }\end{array}$ & $\begin{array}{l}\text { LIRA I.2 mg QD (25I) } \\
\text { LIRA I.8 mg QD (247) } \\
\text { GLIM } 8 \text { mg QD (248) }\end{array}$ & None & $\begin{array}{l}\text { LIRA I.2: }-0.8^{* *} \\
\text { LIRA I.8: }-1 . I^{* * *} \neq \\
\text { GLIM: }-0.5\end{array}$ & $\begin{array}{l}\text { LIRA I.2: }-0.8^{*} \\
\text { LIRA I.8: }-1.4^{* * *} \neq \\
\text { GLIM: }-0.3\end{array}$ & $\begin{array}{l}\text { LIRA I.2: }-2.0^{* * * *} \\
\text { LIRA I.8: }-2.5^{* * *} \\
\text { GLIM: }+1.1\end{array}$ \\
\hline $\begin{array}{l}\text { Garber et } \mathrm{al}^{26} \\
\text { (LEAD-3 Mono; } \\
\text { NCT00294723) }\end{array}$ & $\begin{array}{l}\text { OL extension } \\
\text { of pts with T2D } \\
\text { completing the } \\
\text { LEAD- } 3 \text { core study } \\
\text { ( } 52 \text { weeks) }\end{array}$ & $\begin{array}{l}\text { LIRA I } .2 \mathrm{mg} \text { QD (I I0) } \\
\text { LIRA I .8 mg QD (I I4) } \\
\text { GLIM } 8 \text { mg QD (97) }\end{array}$ & None & $\begin{array}{l}\text { LIRA I.2: }-0.9^{*} \\
\text { LIRA I.8: }-1 . I^{* *} \\
\text { GLIM: }-0.6\end{array}$ & $\begin{array}{l}\text { LIRA I.2: }-1.3^{* *} \\
\text { LIRA I.8: }-1.5^{* * *} \\
\text { GLIM: }-0.3\end{array}$ & $\begin{array}{l}\text { LIRA I.2: }-2.1^{* * *} \\
\text { LIRA I.8: }-2.7^{* * *} \\
\text { GLIM: }+1.1\end{array}$ \\
\hline $\begin{array}{l}\text { Seino et al }{ }^{30} \\
\text { (NCT003937I8) }\end{array}$ & $\begin{array}{l}\mathrm{DB}, \mathrm{DD}, \mathrm{MC} \text {, } \\
\mathrm{PG}, \mathrm{R} \text { in Japanese } \\
\text { pts with T2D } \\
(24 \text { weeks) }\end{array}$ & $\begin{array}{l}\text { LIRA } 0.9 \text { mg QD (272) } \\
\text { GLYB } 2.5 \text { mg/day (I39) }\end{array}$ & None & $\begin{array}{l}\text { LIRA: }-1.9 * * * \\
\text { GLYB: }-1.4\end{array}$ & $\begin{array}{l}\text { LIRA: }-3.6^{* * *} \\
\text { GLYB: }-2.9\end{array}$ & $\begin{array}{l}\text { LIRA: }-0.9 * * * \\
\text { GLYB: }+1.0\end{array}$ \\
\hline $\begin{array}{l}\text { Kaku et al }{ }^{27} \\
(\text { NCT003937I8) }\end{array}$ & $\begin{array}{l}\text { OL extension of } \\
\text { Seino study in } \\
\text { Japanese pts with } \\
\text { T2D (52 weeks) }\end{array}$ & $\begin{array}{l}\text { LIRA } 0.9 \text { mg QD (268) } \\
\text { GLYB } 2.5 \text { mg/day }(132)\end{array}$ & None & $\begin{array}{l}\text { LIRA: }-1.5 \\
\text { GLYB: }-1.0\end{array}$ & $\begin{array}{l}\text { LIRA: }-3.2 \\
\text { GLYB: }-2.5\end{array}$ & $\begin{array}{l}\text { LIRA: }-0.8 \\
\text { GLYB: }+1.0\end{array}$ \\
\hline Thiazolidinediones & & & & & & \\
\hline $\begin{array}{l}\text { DeFronzo et al }{ }^{34} \\
\text { (NCT00I35330) }\end{array}$ & $\begin{array}{l}\text { OL, MC, PG, R } \\
\text { in MET-treated } \\
\text { pts with T2D } \\
\text { (20 weeks) }\end{array}$ & $\begin{array}{l}\text { ExBID I0 } \mu g^{d}(45) \\
\text { ROSI } 4 \mathrm{mg} \text { BID }^{\mathrm{d}}(45) \\
\text { ExBID I0 } \mu \mathrm{g}+\text { ROSI } 4 \mathrm{mg} \\
\operatorname{BID}^{\mathrm{d}}(47)\end{array}$ & MET & $\begin{array}{l}\text { ExBID: }-0.9 \\
\text { ROSI: }-1.0\end{array}$ & $\begin{array}{l}\text { ExBID: }-1.5 \\
\text { ROSI: }-1.8\end{array}$ & $\begin{array}{l}\text { ExBID: }-2.8^{* * *} \\
\text { ROSI: }+1.5\end{array}$ \\
\hline $\begin{array}{l}\text { Xu et a } \mathrm{a}^{35} \\
\text { (CONFIDENCE; } \\
\text { NCTOI I 47627) }\end{array}$ & $\begin{array}{l}\text { OL, MC, PG, R } \\
\text { in treatment- } \\
\text { naive pts with } \\
\text { newly diagnosed } \\
\text { T2D (48 weeks) }\end{array}$ & $\begin{array}{l}\text { ExBID I0 } \mu g^{d}(\mid 42) \\
\text { PIO } 30-45 \text { mg QD (I36) } \\
\text { ILis } 0.2 \mathrm{IU} / \mathrm{kg} \text { BID }^{f}(138)\end{array}$ & None & $\begin{array}{l}\text { ExBID: }-1.8^{* *} \\
\text { PIO: }-1.5\end{array}$ & $\begin{array}{l}\text { ExBID: }-1.9 \\
\text { PIO: }-2.0\end{array}$ & $\begin{array}{l}\text { ExBID: }-3.5^{* * *} \\
\text { PIO: } 0\end{array}$ \\
\hline $\begin{array}{l}\text { Bergenstal et al } \\
\text { (DURATION-2; }^{8} \\
\text { NCT00637273) }\end{array}$ & $\begin{array}{l}\mathrm{DB}, \mathrm{DD}, \mathrm{MC} \\
\mathrm{PG}, \mathrm{R} \text { in pts with } \\
\text { T2D (26 weeks) }\end{array}$ & $\begin{array}{l}\text { ExQW } 2 \text { mg (I60) } \\
\text { SITA } 100 \text { mg QD (I66) } \\
\text { PIO } 45 \text { mg QD (165) }\end{array}$ & MET & $\begin{array}{l}\text { ExQW: }-1.5^{*} \\
\text { PIO: }-1.2\end{array}$ & $\begin{array}{l}\text { ExQW: }-1.8 \\
\text { PIO: }-1.5\end{array}$ & $\begin{array}{l}\text { ExQW: }-2.3^{* * * *} \\
\text { PIO: }+2.8\end{array}$ \\
\hline
\end{tabular}


Table I (Continued)

\begin{tabular}{|c|c|c|c|c|c|c|}
\hline \multirow{2}{*}{$\begin{array}{l}\text { Publications } \\
\text { (acronym/ } \\
\text { ClinicalTrials.gov } \\
\text { record) }\end{array}$} & \multirow[t]{2}{*}{$\begin{array}{l}\text { Study design } \\
\text { (study duration) }\end{array}$} & \multirow[t]{2}{*}{$\begin{array}{l}\text { Treatment (patient } \\
\text { number) }\end{array}$} & \multirow[t]{2}{*}{$\begin{array}{l}\text { Background } \\
\text { therapy }\end{array}$} & \multicolumn{3}{|c|}{$\begin{array}{l}\text { Change from baseline at study end, GLP-IRAs versus } \\
\text { comparator }\end{array}$} \\
\hline & & & & HbAlc (\%) & FG (mmol/L) & BW (kg) \\
\hline $\begin{array}{l}\text { Russell-Jones et al } \\
\text { (DURATION-4; }^{9} \\
\text { NCT00676338) }\end{array}$ & $\begin{array}{l}\mathrm{DB}, \mathrm{MC}, \mathrm{PG}, \mathrm{R} \\
\text { in treatment- } \\
\text { naive pts with } \\
\text { T2D (26 weeks) }\end{array}$ & $\begin{array}{l}\text { ExQW } 2 \text { mg (248) } \\
\text { MET } 2000 \text { mg/day (246) } \\
\text { PIO } 45 \text { mg QD (163) } \\
\text { SITA } 100 \text { mg QD (I63) }\end{array}$ & None & $\begin{array}{l}\text { ExQW: }-1.5 \\
\text { PIO: }-1.6\end{array}$ & $\begin{array}{l}\text { ExQW: }-2.3 \\
\text { PIO: }-2.6\end{array}$ & $\begin{array}{l}\text { ExQW: }-2.0 * * * \\
\text { PIO: }+1.5\end{array}$ \\
\hline $\begin{array}{l}\text { Marre et al }{ }^{33} \\
\text { (LEAD-I SU; } \\
\text { NCT003 I8422) }\end{array}$ & $\begin{array}{l}D B, D D, M C \text {, } \\
P G, R \text { in pts with } \\
\text { T2D treated } \\
\text { with oral GLT } \\
\text { for } \geq 3 \text { months } \\
\text { ( } 26 \text { weeks) }\end{array}$ & $\begin{array}{l}\text { LIRA } 0.6 \mathrm{mg} / \text { day }(233) \\
\text { LIRA I } .2 \mathrm{mg} / \text { day }(228) \\
\text { LIRA I.8 mg/day (234) } \\
\text { ROSI } 4 \mathrm{mg} / \text { day (232) } \\
\text { PBO (I I } 4)\end{array}$ & GLIM & $\begin{array}{l}\text { LIRA 0.6: }-0.6 \\
\text { LIRA I.2: }-1 . I^{* * *} \\
\text { LIRA I.8: }-1 . I^{* * *} \\
\text { ROSI 4: }-0.4\end{array}$ & $\begin{array}{l}\text { LIRA 0.6: }-0.7 \\
\text { LIRA I.2: }-1.6^{* *} \\
\text { LIRA I.8: }-1.6^{* *} \\
\text { ROSI 4: }-0.9\end{array}$ & $\begin{array}{l}\text { LIRA 0.6: }+0.7^{* * * *} \\
\text { LIRA I.2: }+0.3^{* * *} \\
\text { LIRA I.8: }-0.2^{* * *} \\
\text { ROSI 4: }+2.1\end{array}$ \\
\hline
\end{tabular}

Notes: aAfter 12 weeks, LIRA up-titrated to $1.8 \mathrm{mg} /$ day in patients with HbAlc $\geq 7.0 \%$ (53 mmol/mol); bafter $12 \mathrm{meeks}$, GLIM I or $2 \mathrm{mg} /$ day added to regimen of patients with $\mathrm{HbAlc} \geq 7.0 \%$ ( $53 \mathrm{mmol} / \mathrm{mol})$ and FG $>6.1 \mathrm{mmol} / \mathrm{L}$; ${ }^{\mathrm{c}}$ primary end point was the change from baseline in $\mathrm{HbAlc}$ at 26 weeks. Secondary end points included the change from baseline in $\mathrm{HbAlc}$ at 52 weeks, and the change from baseline in FG and BW at 26 and 52 weeks; dadministered at half the named dosage for the first month of the study; edosage adjusted every 4 weeks up to the maximum tolerated dosage according to country-specific labeling information; fdosage was titrated according to self-monitored blood glucose levels. $* P<0.05 ; * * P<0.0$ I; $* * * P \leq 0.00$ I, GLP-IRA versus comparator; $\dagger P<0.0$ I, $\dagger \dagger P \leq 0.000$ I versus baseline (start of extension); $\ddagger P<0.05$ versus LIRA I.2 mg. Abbreviations: AWARD, Assessment of Weekly Administration of Dulaglutide in Diabetes; BID, twice daily; BW, body weight; CO, crossover; CONFIDENCE, Comparison of Glycaemic Control and $\beta$-Cell Function Amongst Newly Diagnosed Patients With Type 2 Diabetes Treated With Exenatide, Insulin or Pioglitazone: A Multicentre Randomized Parallel-Group Study; DB, double blind; DD, double dummy; DPP-4, dipeptidyl peptidase 4; DULA, dulaglutide; DURATION, Diabetes Therapy Utilization: Researching Changes in AIC, Weight and Other Factors Through Intervention With Exenatide Once Weekly; EUREXA, European Exenatide Study; ExBID, exenatide twice daily; ExQW, exenatide once weekly; FG, fasting glucose; GLIM, glimepiride; GLP-IRA, glucagon-like peptide-I receptor agonist; GLT, glucose-lowering therapy; GLYB, glyburide; HbAlc, glycated hemoglobin; ILis, insulin lispro; LEAD, Liraglutide Effect and Action in Diabetes; LIRA, liraglutide; LIXI, lixisenatide; MC, multicenter; MET, metformin; NR, not reported; OL, open label; PBO, placebo; PG, parallel group; PIO, pioglitazone; pts, patients; QD, once daily; QW, once weekly; R, randomized; ROSI, rosiglitazone; SB, single blind; SITA, sitagliptin; T2D, type 2 diabetes; TID, three times a day; TZD, thiazolidinedione; VILD, vildagliptin.

AWARD-3 was similar between the dulaglutide $1.5 \mathrm{mg}$ and metformin groups at 26 and 52 weeks; dulaglutide $0.75 \mathrm{mg}$ recipients lost significantly less weight than patients receiving metformin at both time points.

Minor hypoglycemia was reported in $2.0 \%$ of exenatide QW recipients versus $0.0 \%$ of metformin recipients, while dulaglutide and metformin recipients had similar total hypoglycemia rates $(12.3 \%, 11.1 \%$, and $12.7 \%$ for dulaglutide $1.5 \mathrm{mg}$, dulaglutide $0.75 \mathrm{mg}$, and metformin, respectively). ${ }^{9,21}$ The incidence of gastrointestinal AEs was similar between treatment groups in AWARD- $3,{ }^{21}$ whereas in DURATION-4, nausea was reported in $11.3 \%$ and vomiting in $4.8 \%$ of exenatide-treated patients compared with $3.3 \%$ who experienced vomiting on metformin. ${ }^{9}$

\section{GLP-IRAs versus sulfonylureas}

Nine prospective studies investigated the comparative efficacy of GLP-1RAs and sulfonylureas. ${ }^{22-30}$ One retrospective study was identified: a comparison of liraglutide and glimepiride in outpatients in Italy. ${ }^{31}$

\section{Prospective studies}

Generally, exenatide BID recipients had greater or similar reductions from baseline in $\mathrm{HbA1c}, \mathrm{FG}$, and weight compared with glyburide or glimepiride recipients (Table 1). ${ }^{22-24}$ Furthermore, glycemic control was maintained for a longer duration with exenatide BID compared with glimepiride in the European Exenatide Study (EUREXA). ${ }^{24}$ Compared with glimepiride, liraglutide resulted in greater or similar reductions in $\mathrm{HbA} 1 \mathrm{c}$ and $\mathrm{FG}$, and consistently greater reductions in weight. ${ }^{25,26,28,32}$ Similar results were seen when liraglutide was compared with glyburide, with greater or similar reductions from baseline in $\mathrm{HbA1c}$ and FG; however, weight loss with liraglutide was not consistently greater versus glyburide. ${ }^{27,30}$

Patients receiving GLP-1RAs generally had significantly $(P<0.05)$ lower rates of hypoglycemia than sulfonylureatreated patients; ${ }^{22-30}$ in cases where the $P$ value was not stated, the rates were numerically different in favor of the GLP-1RA. The incidence of gastrointestinal AEs was generally higher with GLP-1RAs compared with sulfonylureas, ${ }^{25-28,30}$ however, one study reported similar incidence of gastrointestinal AEs with exenatide and glibenclamide. ${ }^{22}$ The incidence of treatment withdrawal due to gastrointestinal AEs was also higher with GLP-1RAs compared with sulfonylureas; ${ }^{22-24,32}$ differences in rates of gastrointestinal AEs were seen within 4 weeks in three studies ${ }^{29,30}$ and within 6 months in another study. ${ }^{24}$

\section{Retrospective studies}

In the retrospective analysis, liraglutide produced significantly greater reductions in $\mathrm{HbA} 1 \mathrm{c}$ and $\mathrm{FG}$ from baseline compared with glimepiride (Table $2 ; P<0.001$ ). ${ }^{31}$ 
Table 2 Study details and efficacy results of retrospective studies of GLP-IRAs and oral glucose-lowering therapies or insulin

\begin{tabular}{|c|c|c|c|c|c|c|}
\hline \multirow[t]{2}{*}{ Publications } & \multirow[t]{2}{*}{ Study design (study duration) } & \multirow[t]{2}{*}{$\begin{array}{l}\text { Treatment } \\
\text { (patient number) }\end{array}$} & \multirow[t]{2}{*}{$\begin{array}{l}\text { Background } \\
\text { therapy }\end{array}$} & \multicolumn{3}{|c|}{$\begin{array}{l}\text { Change from baseline at study end, } \\
\text { GLP-IRAs versus comparator }\end{array}$} \\
\hline & & & & HbAlc (\%) & FG (mmol/L) & BW (kg) \\
\hline \multicolumn{7}{|c|}{ Dipeptidyl peptidase-4 inhibitors } \\
\hline Horton et $\mathrm{al}^{18}$ & $\begin{array}{l}\text { Retrospective analysis of adult pts } \\
\text { with T2D using data from the GEC } \\
\text { database (3-12 months) }\end{array}$ & $\begin{array}{l}\text { EXE }(6280) \\
\text { INS }(32,398) \\
\text { SITA }(586 I)\end{array}$ & OADs & $\begin{array}{l}\text { EXE: }-0.5 \\
\text { SITA: }-0.6\end{array}$ & $\begin{array}{l}\text { EXE: }-0.6 \\
\text { SITA: }-0.8\end{array}$ & $\begin{array}{l}\text { EXE: }-3.0 \\
\text { SITA: }-I . I\end{array}$ \\
\hline Montilla et al ${ }^{19}$ & $\begin{array}{l}\text { Retrospective database analysis of } \\
\text { adult pts with T2D in primary care } \\
\text { in Italy (3-12 months) }\end{array}$ & $\begin{array}{l}\text { EXE }(2 I, 064) \\
\text { SITA }(38,8 I I) \\
\text { VILD }(17,989)\end{array}$ & OADs & $\begin{array}{l}\text { EXE: }-1.0 \\
\text { SITA: }-0.9 \\
\text { VILD: }-0.9\end{array}$ & NR & $\begin{array}{l}\text { EXE: } \downarrow 3.5 \% \\
\text { SITA: } \downarrow \\
\sim 1.0 \%-1.5 \% \\
\text { VILD: } \downarrow \\
\sim 1.0 \%-1.5 \%\end{array}$ \\
\hline Nyeland et al ${ }^{20}$ & $\begin{array}{l}\text { Retrospective database analysis of } \\
\text { adult pts with T2D in primary care } \\
\text { in UK ( } 6 \text { months) }\end{array}$ & $\begin{array}{l}\text { LIRA (287) } \\
\text { SITA (278I) }\end{array}$ & OADs & $\begin{array}{l}\text { LIRA: }-0.9 * * \\
\text { SITA: }-0.6\end{array}$ & NR & $\begin{array}{l}\text { LIRA: }-3.8^{* * *} \\
\text { SITA: }-1 . I\end{array}$ \\
\hline \multicolumn{7}{|l|}{ Insulins } \\
\hline Baser et a $\left.\right|^{58}$ & $\begin{array}{l}\text { Retrospective analysis of adult pts } \\
\text { with T2D using data from IMPACT } \\
\text { national managed care database } \\
\text { (I year) }\end{array}$ & $\begin{array}{l}\text { EXE (I958) } \\
\text { IG (38I) }\end{array}$ & OADs & $\begin{array}{l}\text { EXE: }-0.9 \\
\text { IG: }-1.2^{*}\end{array}$ & NR & NR \\
\hline $\begin{array}{l}\text { Bounthavong } \\
\text { et } \mathrm{al}^{59}\end{array}$ & $\begin{array}{l}\text { Retrospective analysis of adult pts } \\
\text { with T2D using data from the VHA } \\
\text { ( } 2 \text { years) }\end{array}$ & $\begin{array}{l}\text { ExBID }(446) \\
\text { IG or IDet }(5 I, 53 I)\end{array}$ & NR & $\begin{array}{l}\text { ExBID: }-0.6 \\
\text { LAI: }-0.7\end{array}$ & NR & NR \\
\hline Dalal et al ${ }^{60}$ & $\begin{array}{l}\text { Retrospective analysis of adult pts } \\
\text { with T2D using data from IMPACT } \\
\text { national managed care database } \\
\text { (I year) }\end{array}$ & $\begin{array}{l}\text { EXE or LIRA (I705) } \\
\text { RAI }(50 \mid 3)\end{array}$ & Basal INS & $\begin{array}{l}\text { GLP-IRA: }-0.6 \\
\text { RAI: }-0.6\end{array}$ & NR & NR \\
\hline Horton et a $\left.\right|^{18}$ & $\begin{array}{l}\text { Retrospective analysis of adult pts } \\
\text { with T2D using data from the GEC } \\
\text { database (3-12 months) }\end{array}$ & $\begin{array}{l}\text { EXE }(6280) \\
\text { INS }(32,398) \\
\text { SITA }(586 I)\end{array}$ & OADs & $\begin{array}{l}\text { EXE: }-0.5 \\
\text { INS: }-1.0\end{array}$ & $\begin{array}{l}\text { EXE: }-0.6 \\
\text { INS: }-1.4\end{array}$ & $\begin{array}{l}\text { EXE: }-3.0 \\
\text { INS: }+0.6\end{array}$ \\
\hline Pawaskar et $\mathrm{a}^{61}$ & $\begin{array}{l}\text { Retrospective analysis of adult pts } \\
\text { with T2D using data from the GEC } \\
\text { database (I year) }\end{array}$ & $\begin{array}{l}\text { EXE }(4494) \\
\text { IG }(5424)\end{array}$ & OADs & $\begin{array}{l}\text { EXE: }-0.6^{*} \\
\text { IG: }-0.4\end{array}$ & NR & $\begin{array}{l}\text { EXE: }-2.6^{*} \\
\text { IG: }-0.2\end{array}$ \\
\hline Pawaskar et al ${ }^{62}$ & $\begin{array}{l}\text { Retrospective analysis of elderly pts } \\
\text { ( } \geq 65 \text { years) with T2D using data } \\
\text { from the GEC database ( } 1 \text { year) }\end{array}$ & $\begin{array}{l}\text { EXE (1023) } \\
\text { IG (2238) }\end{array}$ & OADs & $\begin{array}{l}\text { EXE: }-0.5^{*} \\
\text { IG: }-0.2\end{array}$ & NR & $\begin{array}{l}\text { EXE: }-2.8^{*} \\
\text { IG: }-0.2\end{array}$ \\
\hline $\begin{array}{l}\text { Sudhakaran } \\
\text { et al }{ }^{63}\end{array}$ & $\begin{array}{l}\text { Retrospective analysis of adult pts } \\
\text { with T2D in India ( } 24 \text { weeks) }\end{array}$ & $\begin{array}{l}\text { ExBID } 5-10 \mu g(47) \\
\text { IG QD }(54) \\
\text { NPH QD or BID }(23)\end{array}$ & OADs & $\begin{array}{l}\text { ExBID: }-1.0 \\
\text { IG: }-0.8 \\
\text { NPH: }-0.7\end{array}$ & $\begin{array}{l}\text { ExBID: }-0.5 \\
\text { IG: }-0.8 \\
\text { NPH: }-0.8\end{array}$ & $\begin{array}{l}\text { ExBID: }-1.6 \\
\text { IG: }+1.8 \\
\text { NPH: }+2.3\end{array}$ \\
\hline \multicolumn{7}{|l|}{ Sulfonylureas } \\
\hline Chiefari et al ${ }^{31}$ & $\begin{array}{l}\text { Retrospective analysis in adult out- } \\
\text { pts with T2D in Italy ( } 18 \text { months) }\end{array}$ & $\begin{array}{l}\text { LIRA } 1.8 \text { mg/day (76) } \\
\text { GLIM } 4 \text { mg/day (103) }\end{array}$ & MET & $\begin{array}{l}\text { LIRA: }-1.4^{* * *} \\
\text { GLIM: }-0.4\end{array}$ & $\begin{array}{l}\text { LIRA: }-2 . I^{* * * *} \\
\text { GLIM: }-0.8\end{array}$ & $\begin{array}{l}\text { LIRA: }-4.0^{* * *} \\
\text { GLIM: } 0.0\end{array}$ \\
\hline
\end{tabular}

Notes: $* P<0.05 ; * * P<0.01 ; * * * P \leq 0.001$, GLP-IRA versus comparator.

Abbreviations: BID, twice daily; BW, body weight; ExBID, exenatide twice daily; EXE, exenatide; FG, fasting glucose; GEC, general electric centricity; GLP-IRA, glucagonlike peptide-I receptor agonist; GLIM, glimepiride; HbAIc, glycated hemoglobin; IDet, insulin detemir; IG, insulin glargine; INS, insulin; LAI, long-acting insulin; LIRA, liraglutide; MET, metformin; OAD, oral antidiabetes drug; NPH, neutral protamine Hagedorn insulin; NR, not reported; pts, patients; QD, once daily; RAI, rapid-acting insulin; SITA, sitagliptin; T2D, type 2 diabetes; VHA, Veterans Health Administration; VILD, vildagliptin.

Liraglutide recipients also lost significantly more weight than glimepiride-treated patients $(P<0.001)$. As in the prospective studies, rates of hypoglycemia were lower with liraglutide than glimepiride. ${ }^{31}$ The incidence of gastrointestinal AEs was higher in the liraglutide group compared with the glimepiride group $(P<0.001) .{ }^{31}$

\section{GLP-IRAs versus thiazolidinediones}

Five prospective studies compared GLP-1RAs with thiazolidinediones. ${ }^{8,9,33-35}$

\section{Prospective studies}

Exenatide BID produced similar reductions in $\mathrm{HbA1c}$ to rosiglitazone and significantly greater reductions than pioglitazone $\left(P<0.01\right.$; Table 1). ${ }^{34,35}$ Similar reductions in FG were seen between exenatide BID and rosiglitazone and pioglitazone. Patients receiving exenatide BID had significantly greater weight loss than those receiving thiazolidinediones $(P<0.0001)$; in both studies, exenatide BID recipients had weight loss of $\sim 3 \mathrm{~kg}$, while patients receiving rosiglitazone gained weight and patients receiving pioglitazone had no change in weight. 
Studies of exenatide QW showed that reductions from baseline in $\mathrm{HbAlc}, \mathrm{FG}$, and weight were either greater or similar than with thiazolidinedione comparators (Table 1). ${ }^{8,9}$ In the DURATION-2 study, exenatide QW recipients had significantly greater reductions in $\mathrm{HbAlc}$ versus pioglitazone, while in DURATION-4, reductions in $\mathrm{HbAlc}$ with exenatide QW and pioglitazone were similar; reductions in FG with exenatide QW and pioglitazone were similar. Significant reductions in weight were seen with exenatide QW in DURATION-2 and -4, whereas patients receiving pioglitazone gained weight (as expected in the presence of a sulfonylurea) in both studies ( $P<0.0001$ between groups).

The Liraglutide Effect and Action in Diabetes (LEAD)-1 SU study showed that liraglutide (0.6, 1.2 , and $1.8 \mathrm{mg}$ /day) was generally more effective as a second-line therapy in improving glycemic parameters and weight than rosiglitazone (Table 1). ${ }^{33}$ Liraglutide $1.2 \mathrm{mg}$ and $1.8 \mathrm{mg}$ was associated with significantly greater reductions in $\mathrm{HbA1c}(P<0.0001)$ and FG $(P<0.001)$ than rosiglitazone. Recipients of liraglutide $0.6 \mathrm{mg}$ or $1.2 \mathrm{mg}$ and rosiglitazone gained weight, whereas recipients of liraglutide $1.8 \mathrm{mg}$ lost weight (Table 1); however, the weight gain with the lower dosages of liraglutide was significantly less than that with rosiglitazone, so the differences from baseline in weight were significant between rosiglitazone and all doses investigated $(P<0.0001)$.

Overall, the rate of hypoglycemia seen with GLP1RAs was slightly greater than or similar to that seen with thiazolidinediones. ${ }^{8,933-35}$ The incidence of gastrointestinal AEs was higher with GLP-1RAs compared with thiazolidinediones. ${ }^{8,9,33-35}$ Gastrointestinal AEs led to treatment withdrawal in a higher number of patients in the exenatide group compared with thiazolidinediones in one study. ${ }^{35}$

\section{GLP-IRAs versus insulin products}

Twenty-three prospective studies ${ }^{35-57}$ and seven retrospective studies $^{18,58-63}$ compared GLP-1RAs with insulin products.

\section{Prospective studies}

Four trials compared GLP-1RAs and insulin aspart (Table 3)..$^{38,47,54,55}$ Exenatide studies showed that exenatide BID produced a similar or lesser reduction in $\mathrm{HbA} 1 \mathrm{c}$ and FG than insulin aspart. ${ }^{38,47,55}$ In contrast, exenatide BID recipients lost weight, while those using insulin aspart gained weight. In two of the three exenatide studies, the between-group difference in weight was significant $(P<0.001)$.

In the BEGINTM: VICTOZA ${ }^{\circledR}$ ADD-ON study, liraglutide significantly reduced $\mathrm{HbAlc}$ and weight versus insulin aspart, while reductions in FG were similar between treatments
(Table 3). ${ }^{54}$ When liraglutide $1.8 \mathrm{mg}$ was compared with insulin degludec, the reductions in $\mathrm{HbAlc}$ and FG were similar between groups; patients receiving liraglutide lost weight while patients receiving insulin gained weight. ${ }^{50}$

After 26 weeks of treatment, exenatide QW recipients had a significantly greater reduction from baseline in $\mathrm{HbA} 1 \mathrm{c}$ than insulin detemir recipients and similar reductions in FG. Similar to liraglutide, those receiving exenatide lost weight, while insulin detemir recipients gained weight (betweengroup difference, $P<0.0001$; Table 3). ${ }^{41}$

Twelve studies compared GLP-1RAs with insulin glargine (Table 3). ${ }^{36,37,39,40,42,44} 46,48,51,52,57$ In the exenatide (BID and QW) studies, reductions from baseline in $\mathrm{HbA1c}$ were greater or similar to those with insulin glargine, whereas reductions in FG were similar or smaller (Table 3). Furthermore, a significant difference was generally observed between the weight loss with exenatide and the weight gain with insulin glargine (Table 3).

In the AWARD-4 study, compared with insulin glargine, treatment with dulaglutide for 26 weeks resulted in significantly greater reductions in $\mathrm{HbA} 1 \mathrm{c}$ in patients also receiving insulin lispro and significantly smaller reductions in FG. ${ }^{39}$ Patients receiving dulaglutide $1.5 \mathrm{mg}$ lost weight versus baseline, while recipients of dulaglutide $0.75 \mathrm{mg}$ and insulin glargine gained weight; however, the differences between the dulaglutide and insulin glargine groups were significant, irrespective of dulaglutide dose (Table 3).

The comparisons between liraglutide and insulin glargine in the Efficacy Assessment of Insulin Glargine Versus Liraglutide After Oral Agents Failure (EAGLE) and LEAD-5 studies were inconsistent with regard to $\mathrm{HbAlc}$ and $\mathrm{FG}$; in the EAGLE study, liraglutide resulted in a lesser reduction in $\mathrm{HbA} 1 \mathrm{c}$ and FG than insulin glargine, whereas in the LEAD-5 study, the reduction in HbA1c was greater with liraglutide versus insulin glargine and the reduction in FG was similar between groups. ${ }^{40,57}$ The change in weight was consistent between the two studies, with liraglutide recipients losing weight and insulin glargine recipients gaining weight (Table 3).

Two studies compared exenatide BID with insulin lispro (Table 3). ${ }^{35,43}$ In both studies, exenatide BID and insulin lispro reduced $\mathrm{HbA} 1 \mathrm{c}$ from baseline to a similar degree. Reductions from baseline in FG were greater or similar with exenatide BID versus insulin lispro; exenatide BID treatment consistently resulted in weight loss, while insulin lispro recipients consistently gained weight.

Generally, the rate of hypoglycemia was lower with GLP-1RAs versus insulin products, ${ }^{35,38-40,43,46,47}$ or similar 
Table 3 Study details and efficacy results of prospective (randomized controlled and noninterventional) trials of GLP-IRAs versus insulin products

\begin{tabular}{|c|c|c|c|c|c|c|}
\hline \multirow{2}{*}{$\begin{array}{l}\text { Publications } \\
\text { (acronym/ } \\
\text { ClinicalTrials.gov } \\
\text { record) }\end{array}$} & \multirow[t]{2}{*}{$\begin{array}{l}\text { Study design } \\
\text { (study duration) }\end{array}$} & \multirow[t]{2}{*}{$\begin{array}{l}\text { Treatment } \\
\text { (patient number) }\end{array}$} & \multirow[t]{2}{*}{$\begin{array}{l}\text { Background } \\
\text { therapy }\end{array}$} & \multicolumn{3}{|c|}{$\begin{array}{l}\text { Change from baseline at study end, GLP-IRAs } \\
\text { versus insulin }\end{array}$} \\
\hline & & & & HbAlc (\%) & FG (mmol/L) & BW (kg) \\
\hline \multicolumn{7}{|l|}{ Insulin } \\
\hline $\begin{array}{l}\text { Ostenson et } \mathrm{al}^{56} \\
\text { (CHOICE; } \\
\text { NCT00635492) }\end{array}$ & $\begin{array}{l}M C, O \text { study in pts } \\
\text { with T2D } \\
\text { (1 } 2 \text { months) }\end{array}$ & $\begin{array}{l}\text { ExBID (I096) } \\
\text { INS (I239) }\end{array}$ & OADs & $\begin{array}{l}\text { ExBID: }-1.0 \\
\text { INS: }-1.8\end{array}$ & NR & $\begin{array}{l}\text { ExBID: }-3.3 \\
\text { INS: }+1.9\end{array}$ \\
\hline $\begin{array}{l}\text { Mathieu et al }{ }^{53} \\
\text { (CHOICE; } \\
\text { NCT00635492) }\end{array}$ & $\begin{array}{l}\text { MC, O study in pts } \\
\text { with T2D } \\
\text { ( } 24 \text { months) }\end{array}$ & $\begin{array}{l}\text { ExBID (III4) } \\
\text { INS (I } 274)\end{array}$ & OADs & $\begin{array}{l}\text { ExBID: }-1.0 \\
\text { INS: }-1.7\end{array}$ & NR & $\begin{array}{l}\text { ExBID: }-3.2 \\
\text { INS: }+2.2\end{array}$ \\
\hline \multicolumn{7}{|l|}{ Insulin aspart } \\
\hline $\begin{array}{l}\text { Bergenstal et al }{ }^{38} \\
\text { (NCT00097877) }\end{array}$ & $\begin{array}{l}\text { MC, OL, PG, } R \text { in pts } \\
\text { with T2D ( } 24 \text { weeks) }\end{array}$ & $\begin{array}{l}\text { ExBID 5-I0 } \mu \mathrm{g}(\text { I 24) } \\
\text { Biphasic IAsp } 30 \text { QD } \\
(124) \\
\text { Biphasic IAsp } 30 \text { BID } \\
(124)\end{array}$ & $\begin{array}{l}\text { MET } \\
\text { SU }\end{array}$ & $\begin{array}{l}\text { ExBID: }-1.8 \\
\text { IAsp QD: } \\
-2.3+\dagger \\
\text { IAsp BID: } \\
-2.8+\dagger\end{array}$ & $\begin{array}{l}\text { ExBID: }-1.2 \\
\text { IAsp QD: }-2.9 \dagger \dagger \\
\text { IAsp BID: }-3.5+\dagger\end{array}$ & $\begin{array}{l}\text { ExBID: }-1.9 \\
\text { IAsp QD: }+2.8 \\
\text { IAsp BID: }+4 . \text { I }\end{array}$ \\
\hline $\begin{array}{l}\text { Gallwitz et al }{ }^{47} \\
\text { (NCT00434954) }\end{array}$ & $\begin{array}{l}\text { MC, OL, PG, } R \text { in pts } \\
\text { with T2D ( } 26 \text { weeks) }\end{array}$ & $\begin{array}{l}\text { ExBID 5-10 } \mu g(I 82) \\
\text { Biphasic IAsp } 30 \text { TID } \\
(18 I)\end{array}$ & MET & $\begin{array}{l}\text { ExBID: }-1.0 \\
\text { IAsp: }-1.1\end{array}$ & $N R$ & $\begin{array}{l}\text { ExBID: }-4.1^{* * *} \\
\text { IAsp: }+1.0\end{array}$ \\
\hline $\begin{array}{l}\text { Nauck et al }{ }^{55} \\
(\text { NCT00082407) }\end{array}$ & $\begin{array}{l}\text { MC, OL, PG, R in pts } \\
\text { with T2D ( } 52 \text { weeks) }\end{array}$ & $\begin{array}{l}\text { ExBID 5-10 } \mu \mathrm{g}(253) \\
\text { Biphasic IAsp BID (248) }\end{array}$ & SU & $\begin{array}{l}\text { ExBID: }-1.0 \\
\text { IAsp: }-0.9\end{array}$ & $\begin{array}{l}\text { ExBID: }-1.8 \\
\text { IAsp: }-1.7\end{array}$ & $\begin{array}{l}\text { ExBID: }-2.5^{* * *} \\
\text { IAsp: }+2.9\end{array}$ \\
\hline $\begin{array}{l}\text { Mathieu et al }{ }^{54} \\
\text { (BEGIN: VICTOZA } \\
\text { ADD-ON; } \\
\text { NCT0I38836I) }\end{array}$ & $\begin{array}{l}\text { MC, OL, PG, } R \text { in } \\
\text { insulin-naive pts with } \\
\text { T2D ( } 26 \text { weeks) }\end{array}$ & $\begin{array}{l}\text { LIRA } 0.6-1.8 \mathrm{mg} / \text { day }(88) \\
\text { IAsp QD }(89)^{\mathrm{a}}\end{array}$ & $\begin{array}{l}\text { IDeg } \\
\text { MET }\end{array}$ & $\begin{array}{l}\text { LIRA: }-0.7^{* *} \\
\text { IAsp: }-0.4\end{array}$ & $\begin{array}{l}\text { LIRA: }-0.1 \\
\text { IAsp: }-0.04\end{array}$ & $\begin{array}{l}\text { LIRA: }-2.8^{* * * *} \\
\text { IAsp: }+0.9\end{array}$ \\
\hline \multicolumn{7}{|l|}{ Insulin degludec } \\
\hline $\begin{array}{l}\text { Gough et al }{ }^{50} \\
\text { (DUAL-I; } \\
\text { NCTOI336023) }\end{array}$ & $\begin{array}{l}\text { MC, OL, PG, R in pts } \\
\text { with T2D ( } 26 \text { weeks) }\end{array}$ & $\begin{array}{l}\text { IDeg/LIRA QD (834) } \\
\text { LIRA 0.6-I.8 mg QD } \\
(4 \mid 5) \\
\text { IDeg QD (4I4) }\end{array}$ & $\begin{array}{l}\text { MET } \\
\pm \mathrm{PIO}\end{array}$ & $\begin{array}{l}\text { LIRA: }-1.3 \\
\text { IDeg: }-1.4\end{array}$ & $\begin{array}{l}\text { LIRA: }-1.8 \\
\text { IDeg: }-3.6\end{array}$ & $\begin{array}{l}\text { LIRA: }-3.0 \\
\text { IDeg: }+1.6\end{array}$ \\
\hline $\begin{array}{l}\text { Gough et } \mathrm{al}^{49} \\
\text { (DUAL-I; } \\
\text { NCT0I336023) }\end{array}$ & $\begin{array}{l}\text { Extension of DUAL-I } \\
\text { in pts with T2D } \\
\text { ( } 52 \text { weeks) }\end{array}$ & $\begin{array}{l}\text { IDeg/LIRA QD (665) } \\
\text { LIRA 0.6-I.8 mg QD } \\
(3 \mid 3) \\
\text { IDeg QD (333) }\end{array}$ & $\begin{array}{l}\text { MET } \\
\pm \mathrm{PIO}\end{array}$ & $\begin{array}{l}\text { LIRA: }-1.2 \\
\text { IDeg: }-1.4\end{array}$ & $\begin{array}{l}\text { LIRA: }-1.7 \\
\text { IDeg: }-3.4\end{array}$ & $\begin{array}{l}\text { LIRA: }-3.0 \\
\text { IDeg: }+2.3\end{array}$ \\
\hline Insulin detemir & & & & & & \\
\hline $\begin{array}{l}\text { Davies et } \mathrm{al}^{41} \\
\text { (NCT0I003 I84) }\end{array}$ & $\begin{array}{l}\text { MC, OL, PG, R in pts } \\
\text { with T2D ( } 26 \text { weeks) }\end{array}$ & $\begin{array}{l}\text { ExQW } 2 \text { mg (IIII) } \\
\text { IDet QD or BID (I05) }\end{array}$ & $\begin{array}{l}\text { MET } \\
\pm S U\end{array}$ & $\begin{array}{l}\text { ExQW: }-1.3^{* * *} \\
\text { IDet: }-0.9\end{array}$ & $\begin{array}{l}\text { ExQW: }-2.3 \\
\text { IDet: }-2.4\end{array}$ & $\begin{array}{l}\text { ExQW: }-2.7 * * * \\
\text { IDet: }+0.8\end{array}$ \\
\hline Insulin glargine & & & & & & \\
\hline $\begin{array}{l}\text { Barnett et al }{ }^{37} \\
(\text { NCT00099619) }\end{array}$ & $\begin{array}{l}C O, M C, O L, R \text { in } \\
\text { pts with T2D } \\
(2 \times 16 \text { weeks })\end{array}$ & $\begin{array}{l}\text { ExBID 5-10 } \mu g / I G \text { QD } \\
(68) \\
\text { IG QD/ExBID 5-10 } \mu g \\
(70)\end{array}$ & $\begin{array}{l}\text { MET (55. } \% \text { ) } \\
\text { SU (44.9\%) }\end{array}$ & $\begin{array}{l}\text { ExBID: }-1.4 \\
\text { IG: }-1.4\end{array}$ & $\begin{array}{l}\text { ExBID: }-2.9 \\
\text { IG: }-4.1+\dagger\end{array}$ & $\begin{array}{l}\text { ExBID: }-1.6^{* * *} \\
\text { IG: }+0.6\end{array}$ \\
\hline Davies et al ${ }^{42}$ (HEELA) & $\begin{array}{l}\text { MC, OL, PG, R in pts } \\
\text { with T2D ( } 26 \text { weeks) }\end{array}$ & $\begin{array}{l}\text { ExBID 5-10 } \mu \mathrm{g}(1 \mathrm{I}) \\
\text { IG QD }(117)\end{array}$ & $\begin{array}{l}\text { MET } \\
\text { SU } \\
\text { TZD }\end{array}$ & $\begin{array}{l}\text { ExBID: }-1.3 \\
\text { IG: }-1.3\end{array}$ & $\begin{array}{l}\text { ExBID: }-2 . I \\
\text { IG: }-3.6+\dagger\end{array}$ & $\begin{array}{l}\text { ExBID: }-2.7^{* * *} \\
\text { IG: }+3.0\end{array}$ \\
\hline $\begin{array}{l}\text { Diamant et al }{ }^{46} \\
\text { (DURATION-3; } \\
\text { NCT0064I056) }\end{array}$ & $\begin{array}{l}\text { MC, OL, PG, R in pts } \\
\text { with T2D ( } 26 \text { weeks) }\end{array}$ & $\begin{array}{l}\text { ExQW } 2 \text { mg (233) } \\
\text { IG QD (223) }\end{array}$ & $\begin{array}{l}\text { MET } \\
\pm S U\end{array}$ & $\begin{array}{l}\text { ExQW: }-1.5^{*} \\
\text { IG: }-1.3\end{array}$ & $\begin{array}{l}\text { ExQW: }-2 . I \\
\text { IG: }-2.8+\dagger\end{array}$ & $\begin{array}{l}\text { ExQW: }-2.6^{* * *} \\
\text { IG: }+1.4\end{array}$ \\
\hline $\begin{array}{l}\text { Diamant et } \mathrm{al}^{45} \\
\text { (DURATION-3 } \\
\text { extension; } \\
\text { NCT0064I056) }\end{array}$ & $\begin{array}{l}\text { OL extension of } \\
\text { DURATION-3 in pts } \\
\text { with T2D ( } 84 \text { weeks) }\end{array}$ & $\begin{array}{l}\text { ExQW } 2 \text { mg (233) } \\
\text { IG QD (223) }\end{array}$ & $\begin{array}{l}\text { MET } \\
\pm S U\end{array}$ & $\begin{array}{l}\text { ExQW: }-1.2 * \\
\text { IG: }-1.0\end{array}$ & $\begin{array}{l}\text { ExQW: }-2.4 \\
\text { IG: }-3.0 \dagger\end{array}$ & $\begin{array}{l}\text { ExQW: }-2 . I^{* * *} \\
\text { IG: }+2.4\end{array}$ \\
\hline $\begin{array}{l}\text { Diamant et a }{ }^{44} \\
\text { (DURATION-3 } \\
\text { extension; } \\
\text { NCT0064I056) }\end{array}$ & $\begin{array}{l}\text { OL extension of } \\
\text { DURATION-3 in pts } \\
\text { with T2D } \\
\text { (I56 weeks) }\end{array}$ & $\begin{array}{l}\text { ExQW } 2 \text { mg (194) } \\
\text { IG QD (196) }\end{array}$ & $\begin{array}{l}\text { MET } \\
\pm S U\end{array}$ & $\begin{array}{l}\text { ExQW: }-1.0^{*} \\
\text { IG: }-0.8\end{array}$ & $\begin{array}{l}\text { ExQW: }-1.7 \\
\text { IG: }-2.7 \dagger \dagger\end{array}$ & $\begin{array}{l}\text { ExQW: }-2.5^{* * *} \\
\text { IG: }+2.0\end{array}$ \\
\hline
\end{tabular}


Table 3 (Continued)

\begin{tabular}{|c|c|c|c|c|c|c|}
\hline \multirow{2}{*}{$\begin{array}{l}\text { Publications } \\
\text { (acronym/ } \\
\text { ClinicalTrials.gov } \\
\text { record) }\end{array}$} & \multirow[t]{2}{*}{$\begin{array}{l}\text { Study design } \\
\text { (study duration) }\end{array}$} & \multirow[t]{2}{*}{$\begin{array}{l}\text { Treatment } \\
\text { (patient number) }\end{array}$} & \multirow[t]{2}{*}{$\begin{array}{l}\text { Background } \\
\text { therapy }\end{array}$} & \multicolumn{3}{|c|}{$\begin{array}{l}\text { Change from baseline at study end, GLP-I RAs } \\
\text { versus insulin }\end{array}$} \\
\hline & & & & HbAlc (\%) & FG (mmol/L) & BW (kg) \\
\hline $\begin{array}{l}\text { Heine et } \mathrm{al}^{5 \mathrm{I}} \\
(\mathrm{NCT} 0008238 \mathrm{I})\end{array}$ & $\begin{array}{l}\text { MC, OL, PG, R in pts } \\
\text { with T2D ( } 26 \text { weeks) }\end{array}$ & $\begin{array}{l}\text { ExBID } 5-10 \mu g(282) \\
\text { IG QD }(267)\end{array}$ & $\begin{array}{l}\text { MET } \\
\text { SU }\end{array}$ & $\begin{array}{l}\text { ExBID: }-I . I \\
\text { IG: }-I . I\end{array}$ & $\begin{array}{l}\text { ExBID: }-1.4 \\
\text { IG: }-2.9+\dagger\end{array}$ & $\begin{array}{l}\text { ExBID: }-2.3 \\
\text { IG: }+1.8\end{array}$ \\
\hline $\begin{array}{l}\text { Inagaki et al }{ }^{52} \\
\text { (NCT00935532) }\end{array}$ & $\begin{array}{l}\text { MC, OL, PG, R in } \\
\text { Japanese pts with } \\
\text { T2D ( } 26 \text { weeks })\end{array}$ & $\begin{array}{l}\text { ExQW } 2 \text { mg (2I5) } \\
\text { IG QD (2I2) }\end{array}$ & $\begin{array}{l}\text { BG } \\
\pm T Z D\end{array}$ & $\begin{array}{l}\text { ExQW: }-\mathrm{I} . \mathrm{I}^{* * *} \\
\mathrm{IG}:-0.7\end{array}$ & $\begin{array}{l}\text { ExQW: }-2.6 \\
\text { IG: }-2.3\end{array}$ & $\begin{array}{l}\text { ExQW: }-1.7^{* * *} \\
\text { IG: }+0.3\end{array}$ \\
\hline $\begin{array}{l}\text { Araki et al }{ }^{36} \\
\text { (NCTOI584232) }\end{array}$ & $\begin{array}{l}\text { MC, OL, PG, R in } \\
\text { Japanese pts with } \\
\text { T2D ( } 26 \text { weeks })\end{array}$ & $\begin{array}{l}\text { DULA } 0.75 \mathrm{mg} \text { QW } \\
(\mathrm{I} 8 \mathrm{I}) \\
\text { IG QD (I80) }\end{array}$ & $\begin{array}{l}\mathrm{BG} \\
\pm \mathrm{SU}\end{array}$ & $\begin{array}{l}\text { DULA } 0.75:-1.4^{* * *} \\
\text { IG: }-0.9\end{array}$ & $\begin{array}{l}\text { DULA } 0.75:-1.9 \\
\text { IG: }-2.1\end{array}$ & $\begin{array}{l}\text { DULA } 0.75: \\
-0.5^{* * *} \\
\text { IG: }+0.9\end{array}$ \\
\hline $\begin{array}{l}\text { Blonde et } \mathrm{al}^{39} \\
\text { (AWARD-4; } \\
\text { NCT0I I9I268) }\end{array}$ & $\begin{array}{l}\text { MC, OL, PG, R in pts } \\
\text { with T2D } \\
\left(52 \text { weeks }^{b}\right)\end{array}$ & $\begin{array}{l}\text { DULA } 0.75 \mathrm{mg} \text { QW } \\
(293) \\
\text { DULA I.5 mg QW (295) } \\
\text { IG QD (296) }\end{array}$ & $\begin{array}{l}\text { ILis } \\
\text { MET }\end{array}$ & $\begin{array}{l}\text { At } 26 \text { weeks } \\
\text { DULA } 0.75:-1.6^{*} \\
\text { DULA I.5: }-1.6^{* *} \\
\text { IG: }- \text { I.4 }\end{array}$ & $\begin{array}{l}\text { At } 26 \text { weeks } \\
\text { DULA } 0.75:-0.2 \\
\text { DULA I.5: }-0.3 \\
\text { IG: }- \text { I. } 6+\dagger\end{array}$ & $\begin{array}{l}\text { At } 26 \text { weeks } \\
\text { DULA } 0.75: \\
+0.2^{* * *} \\
\text { DULA I.5: } \\
-0.9 * * * \\
\text { IG: }+2.3\end{array}$ \\
\hline $\begin{array}{l}\text { D'Alessio et } \mathrm{al}^{40} \\
\text { (EAGLE) }\end{array}$ & $\begin{array}{l}\text { MC, OL, PG, R in pts } \\
\text { with T2D ( } 24 \text { weeks) }\end{array}$ & $\begin{array}{l}\text { LIRA 0.6-I.8 mg QD } \\
(489) \\
\text { IG QD (489) }\end{array}$ & $\begin{array}{l}\text { MET } \\
\pm \text { SU }\end{array}$ & $\begin{array}{l}\text { LIRA: }-1.8 \\
\text { IG: }-1.9 *\end{array}$ & $\begin{array}{l}\text { LIRA: }-2.4 \\
\text { IG: }-2.8+\dagger\end{array}$ & $\begin{array}{l}\text { LIRA: }-3.0 * * * \\
\text { IG: }+2.0\end{array}$ \\
\hline $\begin{array}{l}\text { Giorgino et al }{ }^{48} \\
\text { (AWARD-2; } \\
\text { NCT01075282) }\end{array}$ & $\begin{array}{l}\text { MC, OL, PG, R in pts } \\
\text { with T2D } \\
(78 \text { weeks })\end{array}$ & $\begin{array}{l}\text { DULA } 0.75 \mathrm{mg} \text { QW } \\
(272) \\
\text { DULA I.5 mg QW (273) } \\
\text { IG QD (262) }\end{array}$ & $\begin{array}{l}\text { MET } \\
\text { GLIM }\end{array}$ & $\begin{array}{l}\text { At } 52 \text { weeks } \\
\text { DULA 0.75: } \\
-0.8 * * * \\
\text { DULA I.5: } \\
-I . I^{* * *} \\
\text { IG: }-0.6\end{array}$ & $\begin{array}{l}\text { At } 52 \text { weeks } \\
\text { DULA } 0.75:-0.9 \\
\text { DULA I.5: }-1.5 \\
\text { IG: }-1.8+\dagger^{\mathrm{d}}\end{array}$ & $\begin{array}{l}\text { At } 52 \text { weeks } \\
\text { DULA 0.75: } \\
-I .3 * * * \\
\text { DULA I.5: } \\
-I .9 * * * \\
\text { IG: + I.4 }\end{array}$ \\
\hline $\begin{array}{l}\text { Russell-Jones } \\
\text { et } \mathrm{al}^{57} \text { (LEAD-5; } \\
\text { NCT0033 I85I) }\end{array}$ & $\begin{array}{l}\text { MC, OL, PG, R in pts } \\
\text { with T2D ( } 26 \text { weeks) }\end{array}$ & $\begin{array}{l}\text { LIRA I.8 mg QD (230) } \\
\text { IG QD (232) } \\
\text { PBO (I I4) }\end{array}$ & $\begin{array}{l}\text { MET } \\
\text { GLIM }\end{array}$ & $\begin{array}{l}\text { LIRA: }-I .3^{* *} \\
\text { IG: }-I . I\end{array}$ & $\begin{array}{l}\text { LIRA: }-1.6 \\
\text { IG: }-I .8\end{array}$ & $\begin{array}{l}\text { LIRA: }-1.8^{* * * *} \\
\text { IG: }+1.6\end{array}$ \\
\hline Insulin lispro & & & & & & \\
\hline $\begin{array}{l}\text { Diamant et } \mathrm{al}^{43} \\
\text { (NCT0096066I) }\end{array}$ & $\begin{array}{l}\text { MC, OL, PG, R in pts } \\
\text { with T2D ( } 30 \text { weeks) }\end{array}$ & $\begin{array}{l}\text { ExBID } 5-10 \mu g(3 \mid 5) \\
\text { ILis TID }(3 \mid 2)\end{array}$ & $\begin{array}{l}\text { MET } \\
\text { IG }\end{array}$ & $\begin{array}{l}\text { ExBID: }-I . I \\
\text { ILis: }-I . I\end{array}$ & $\begin{array}{l}\text { ExBID: }-0.5^{* *} \\
\text { ILis: }+0.2\end{array}$ & $\begin{array}{l}\text { ExBID: }-2.5^{* * *} \\
\text { ILis: }+2.1\end{array}$ \\
\hline $\begin{array}{l}\text { Xu et al }{ }^{35} \\
\text { (CONFIDENCE; } \\
\text { NCTOI I47627) }\end{array}$ & $\begin{array}{l}\text { MC, OL, PG, R in } \\
\text { treatment-naive pts } \\
\text { with newly diagnosed } \\
\text { T2D ( } 48 \text { weeks) }\end{array}$ & 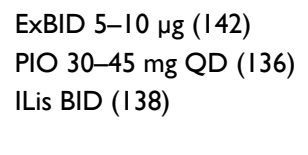 & None & $\begin{array}{l}\text { ExBID: }-1.8 \\
\text { ILis: }-I .7\end{array}$ & $\begin{array}{l}\text { ExBID: }-1.9 \\
\text { ILis: }-1.6\end{array}$ & $\begin{array}{l}\text { ExBID: }-3.5^{* * *} \\
\text { ILis: }+1.0\end{array}$ \\
\hline
\end{tabular}

Notes: ${ }^{\mathrm{a}}$ Treatment arms were also compared with a nonrandomized group of patients who received IDeg alone $(\mathrm{n}=236)$; ${ }^{\mathrm{b}} \mathrm{primary}$ end point was change from baseline in $\mathrm{HbAlc}$ at 26 weeks; ' primary end point was change from baseline in HbAlc at 52 weeks; dsignificant difference applies to IG QD versus DULA 0.75 mg QW comparison only. ${ }^{*} P<0.05 ; * * P<0.01$; $* * * P \leq 0.00$ I, GLP-IRA versus INS comparator; $\dagger P<0.01 ; \dagger+P \leq 0.00$ I, INS comparator versus GLP-IRA.

Abbreviations: AWARD, Assessment of Weekly Administration of Dulaglutide in Diabetes; BG, biguanide; BID, twice daily; BW, body weight; CHOICE, Changes to Treatment and Outcomes in Patients With Type 2 Diabetes Initiating Injectable Therapy; CO, crossover; CONFIDENCE, Comparison of Glycaemic Control and $\beta$-Cell Function Amongst Newly Diagnosed Patients With Type 2 Diabetes Treated With Exenatide, Insulin or Pioglitazone: A Multicentre Randomized Parallel-Group Study; DUAL, Dual Action of Liraglutide and Insulin Degludec in Type 2 Diabetes; DULA, dulaglutide; DURATION, Diabetes Therapy Utilization: Researching Changes in AIC, Weight and Other Factors Through Intervention With Exenatide Once Weekly; EAGLE, Efficacy Assessment of Insulin Glargine versus Liraglutide After Oral Agent Failure; ExBID, exenatide twice daily; ExQW, exenatide once weekly; FG, fasting glucose; GLIM, glimepiride; GLP-IRA, glucagon-like peptide-I receptor agonist; HbAIc, glycated hemoglobin; HEELA, Helping Evaluate Exenatide in Patients With Diabetes Compared With Long-Acting Insulin; IAsp, insulin aspart; IDeg, insulin degludec; IDet, insulin detemir; IG, insulin glargine; ILis, insulin lispro; INS, insulin; LEAD, Liraglutide Effect and Action in Diabetes; LIRA, liraglutide; MC, multicenter; MET, metformin; NR, not reported; O, observational; OAD, oral antidiabetes drug; OL, open label; PBO, placebo; PG, parallel group; PIO, pioglitazone; pts, patients; QD, once daily; QW, once weekly; R, randomized; SU, sulfonylurea; T2D, type 2 diabetes; TID, three times daily; TZD, thiazolidinedione.

between groups. ${ }^{41,55,57}$ Overall, the incidence of gastrointestinal AEs among GLP-1RA-treated patients was higher than in insulin-treated patients; ${ }^{37-42,44-47,50-52,54,55,57}$ gastrointestinal AEs were generally observed on initiation of treatment with GLP-1RAs ${ }^{39,44,45,47,50,57}$ and sometimes led to treatment withdrawal. ${ }^{37,39,42,47,52}$

\section{Retrospective studies}

Seven retrospective studies compared GLP-1RAs and insulin products (Table 2), ${ }^{18,58-63}$ with similar results to the prospective studies; the reduction in HbAlc was greater with GLP1RAs than insulin in most studies. Where reported, reductions from baseline in FG were not statistically different between 
groups. In studies in which the change in weight was reported, GLP-1RA treatment resulted in weight loss, while insulin treatment resulted in weight gain.

Where reported, the incidence of hypoglycemia was either similar between GLP-1RA and insulin treatment groups or lower in those receiving GLP-1RAs. ${ }^{58,60-63}$ Treatment-related nausea was reported in 10 patients in the exenatide group in one study and did not lead to treatment discontinuation. ${ }^{63}$

\section{Effect of GLP-I RAs versus other glucose-lowering therapies on cardiovascular risk factors}

The comparative studies identified by the literature search reported only limited information on cardiovascular risk factors and did not assess cardiovascular outcomes.

\section{GLP-IRAs versus DPP-4is}

Of the 10 prospective trials of GLP-1RAs and DPP-4is, all reported effects on blood pressure (BP) and/or lipid cardiovascular risk factors (Table S1). ${ }^{7-16}$ Two retrospective studies comparing GLP-1RAs with DPP-4is also reported changes in cardiovascular risk factors (Table S2). ${ }^{18,20}$ Changes in both diastolic BP and systolic BP (SBP) were variable for GLP1RAs and DPP-4is, and changes in lipids were generally not significantly different between treatment groups. However, in AWARD-5, dulaglutide $1.5 \mathrm{mg}$ resulted in a significantly greater reduction in low-density lipoprotein cholesterol compared with sitagliptin ${ }^{10}$ (Table S1).

\section{GLP-IRAs versus metformin}

Two studies reported data on cardiovascular risk factors after treatment with GLP-1RAs compared with metformin, neither of which showed any clinically significant between-group differences in cardiovascular risk factors (Table S1).$^{9,21}$

\section{GLP-IRAs versus sulfonylureas}

Seven of the nine studies comparing GLP-1RAs with sulfonylureas reported data on cardiovascular risk factors (Table S1) ${ }^{24-28,30,32}$ along with one retrospective study (Table S2).${ }^{31}$ In general, results indicated that the GLP-1RAs were associated with greater reductions in SBP than the sulfonylureas, with numerical reductions in various lipids observed in several studies comparing GLP-1RAs with sulfonylureas.

\section{GLP-IRAs versus thiazolidinediones}

All five prospective studies comparing GLP-1RAs with thiazolidinediones reported reductions from baseline in BP or fasting lipids (Table S1) $)^{8,9,33-35}$ Exenatide significantly reduced measures of cholesterol versus thiazolidinediones in three of these studies. ${ }^{8,34,35}$

\section{GLP-IRAs versus insulin}

Two of four trials comparing GLP-1RAs and insulin aspart reported changes in cardiovascular risk factors (Table S3 $){ }^{54,55}$ One trial reported a significantly smaller increase in highdensity lipoprotein cholesterol with exenatide BID versus insulin aspart. ${ }^{55}$

Eight of ten prospective studies and two extension studies comparing GLP-1RAs with insulin glargine reported changes in cardiovascular risk factors (Table S3). ${ }^{36,39,40,42,44-46,48,52,57}$ Overall, numerical reductions in SBP were observed in most of these studies, with greater reductions generally seen with GLP-1RAs than for insulin glargine; significant differences between treatments for fasting lipids also generally favored GLP-1RAs.

Exenatide BID was compared with insulin lispro in two studies (Table S3). ${ }^{35,43}$ No significant differences between treatment groups were reported for changes in BP or lipids.

Of the seven retrospective studies comparing GLP-1RAs and insulin products, four reported data on cardiovascular risk factors (Table S2). ${ }^{18,61-63}$ Numerical reductions in BP and lipid measures were observed across exenatide and insulin therapies.

\section{Discussion}

GLP-1RAs represent an effective therapeutic option for patients with T2D. Currently, multiple studies provide data on the efficacy of approved GLP-1RAs in T2D (Figure 2). Although their glycemic efficacy is well established versus multiple classes of agents, including insulin, albeit with a notable lack of comparative studies versus sodium-glucose cotransporter 2 inhibitors, GLP-1RAs have additional benefits of modest weight loss and a favorable tolerability profile (Figure 3). ${ }^{64}$ These beneficial effects of GLP-1RAs should be considered in context with the increased frequency of nausea with GLP-1RAs, which may limit adherence, and the need for self-injection on a once- or twice-daily or weekly basis.

The efficacy of GLP-1RAs is generally greater than DPP4is, owing to the supraphysiological concentrations of GLP-1 after administration of the former. ${ }^{65}$ This effect on incretin also accounts for the greater weight loss experienced by patients who receive GLP-1RAs versus the neutral weight effects produced by DPP-4is. ${ }^{65,66}$ Glycemic control was also mostly 


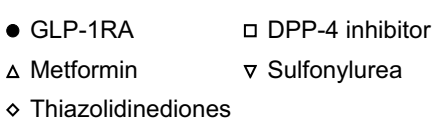

A

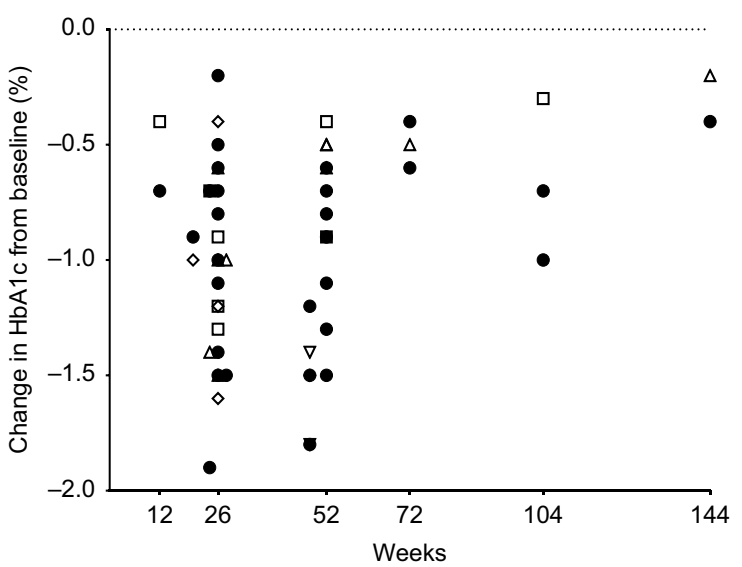

$\mathbf{B}$

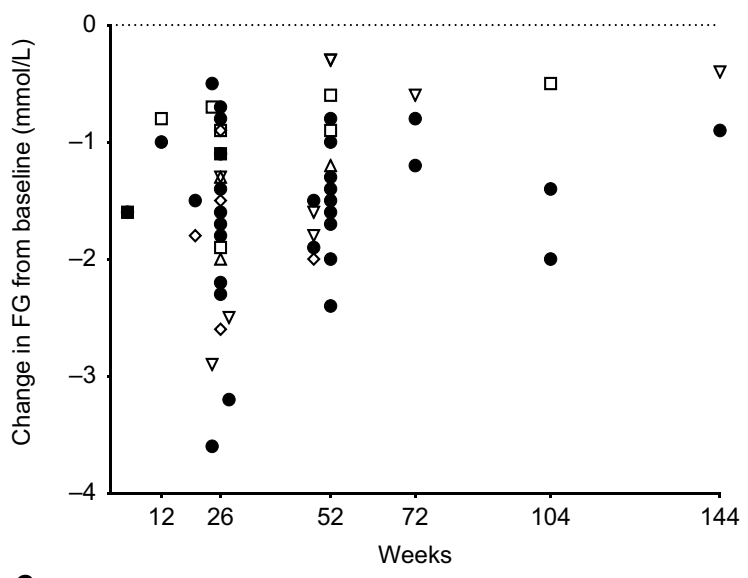

C

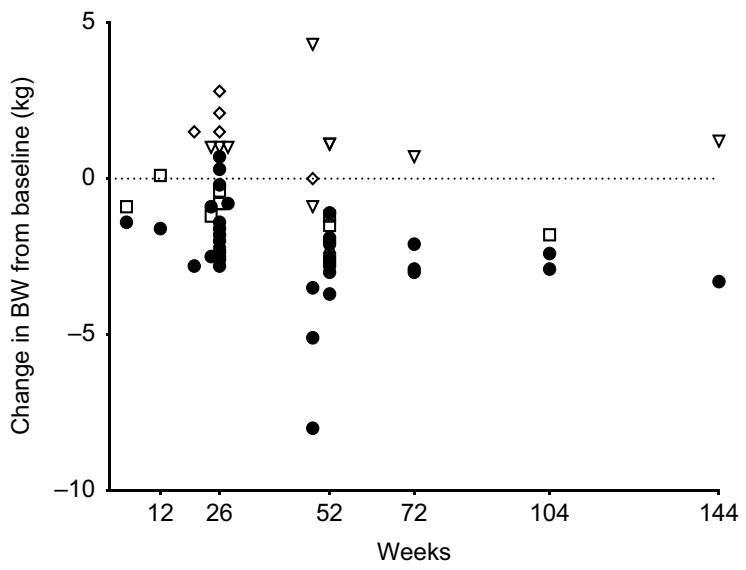

D

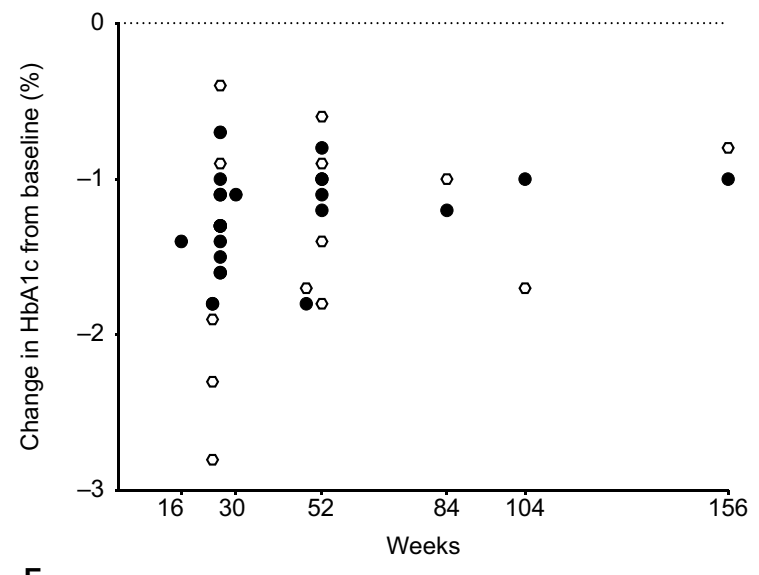

E

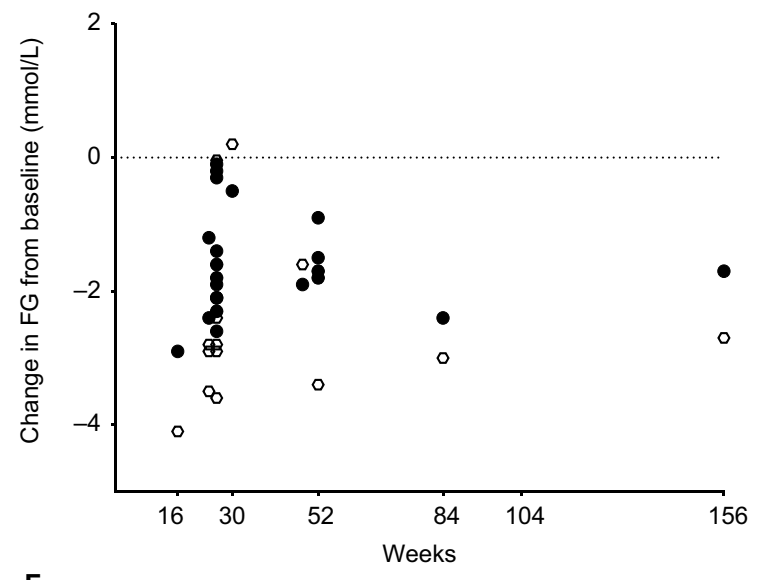

$\mathbf{F}$

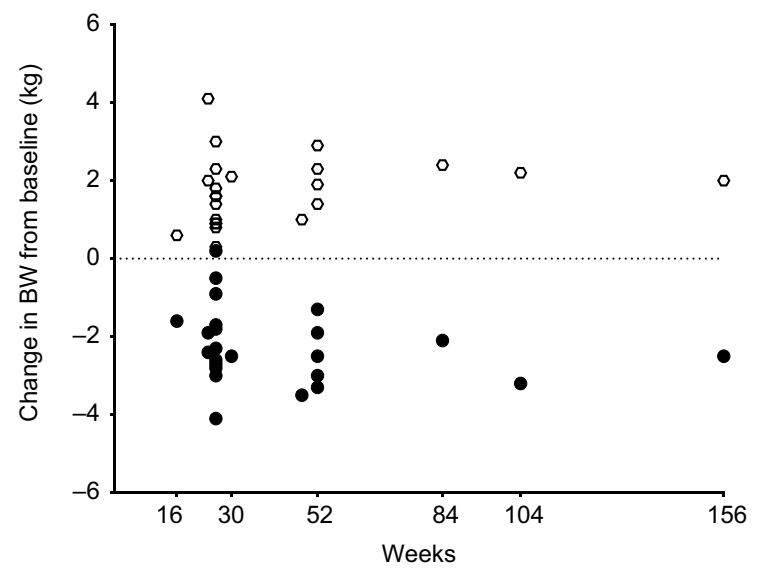

Figure 2 Efficacy of glucose-lowering treatments in type 2 diabetes. Changes from baseline in (A) glycated hemoglobin (HbAIc), (B) fasting glucose (FG), and (C) body weight (BW) in prospective studies comparing glucagon-like peptide-I receptor agonists (GLP-IRAs) with other oral glucose-lowering therapies and changes from baseline in (D) $\mathrm{HbAlc}$, (E) FG, and (F) BW in prospective studies comparing GLP-IRAs with insulin products.

Abbreviation: DPP-4, dipeptidyl peptidase-4.

similar to that with insulin, largely driven by improvements in postprandial glucose with the short-acting GLP-1RAs, with similar effects on FG as insulin. However, real-world evidence suggests similar glycemic reductions between GLP-1RAs and insulin and within the GLP-1RA class. A real-world study in patients with $\mathrm{T} 2 \mathrm{D}$ reported that addition of exenatide BID to basal insulin was as effective as addition of mealtime insulin in reducing $\mathrm{HbA} 1 \mathrm{c}$ levels in these patients, with significant 


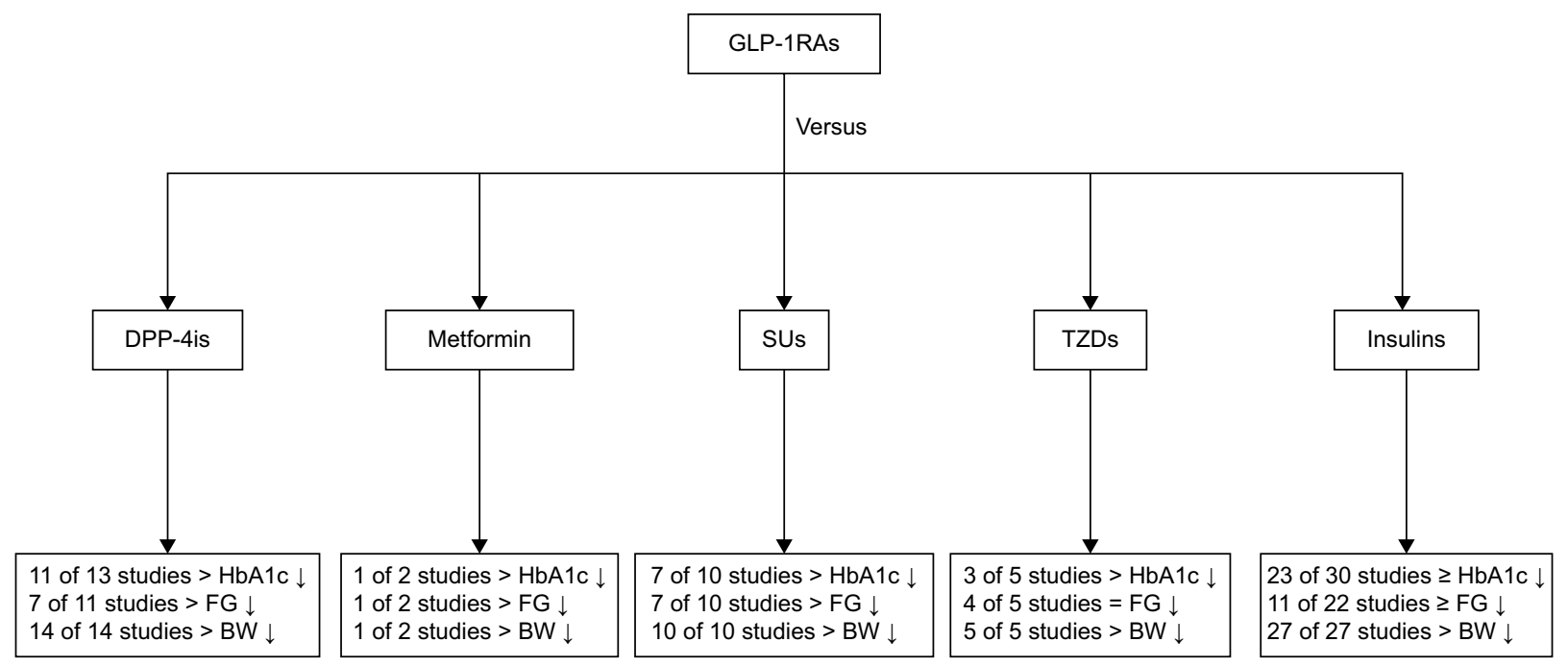

Figure 3 Comparison of efficacy of GLP-IRAs with other glucose-lowering treatments in type 2 diabetes. General trends in glycemic parameters and body weight (BW) in comparative trials of glucagon-like peptide-I receptor agonists (GLP-IRAs) and other glucose-lowering therapies. The total number of studies includes studies that reported these parameters.

Abbreviations: DPP-4i, dipeptidyl peptidase-4 inhibitor; FG, fasting glucose; HbAlc, glycated hemoglobin; SU, sulfonylurea; TZD, thiazolidinedione.

reductions in weight $(P<0.01)$ and hypoglycemia $(P<0.03)$ compared with mealtime insulin. ${ }^{67}$

Data comparing the effects of GLP-1RAs and other classes of glucose-lowering therapy on cardiovascular risk factors were limited but showed favorable effects on BP and lipid levels. Increases in heart rate have been observed with GLP1RAs in clinical trials, although the underlying mechanisms and clinical relevance of these increases have yet to be established. ${ }^{68}$ The size and duration of increases in mean 24-hour heart rate vary between GLP-1RAs, ranging from transient (1-12 hours) increases of $1-3$ beats per minute (bpm) with the short-acting GLP-1RAs exenatide BID and lixisenatide to more prolonged increases during treatment with longer-acting GLP-1RAs (3-4 bpm with exenatide QW or dulaglutide and 6-10 bpm with liraglutide or albiglutide). ${ }^{68}$ Data from cardiovascular outcome trials indicate that the observed increases in heart rate were not associated with an adverse effect on cardiovascular outcomes in patients with T2D. ${ }^{5,6,68,69}$

Limited clinical data are available regarding the effects of the various GLP-1RAs on cardiovascular outcomes, and none of these studies compared a GLP-1RA with another class of glucose-lowering therapy. The first published study with prospective outcomes data was the Evaluation of Lixisenatide in Acute Coronary Syndrome (ELIXA) trial, ${ }^{70}$ which found no significant difference in rates of cardiovascular events with lixisenatide versus placebo. ${ }^{6}$ A similar study investigating the effects of liraglutide on cardiovascular outcomes, the Liraglutide Effect and Action in Diabetes: Evaluation of Cardiovascular Outcome Results (LEADER) trial, showed improved effects of liraglutide on cardiovascular outcomes versus placebo. ${ }^{5}$ Finally, the Trial to Evaluate Cardiovascular and Other Long-term Outcomes With Semaglutide in Subjects With Type 2 Diabetes (SUSTAIN-6), which evaluated the effect of semaglutide (added on to standard care) on cardiovascular outcomes, demonstrated the noninferiority of semaglutide to placebo, with a significant reduction in cardiovascular death, nonfatal stroke, or nonfatal myocardial infarction in the semaglutide group. ${ }^{69}$

Results of the EXenatide Study of Cardiovascular Event Lowering (EXSCEL) trial, evaluating the effect of exenatide QW on major adverse cardiovascular events (MACE) when given in addition to usual care $;{ }^{71}$ the Researching Cardiovascular Events With a Weekly Incretin in Diabetes (REWIND) study, evaluating the effects of dulaglutide on MACE; $;^{72}$ and the HARMONY Outcomes study, evaluating the effects of albiglutide on MACE ${ }^{73}$ are all awaited and are expected to help contribute to the existing evidence regarding the impact of the GLP-1RAs on cardiovascular outcomes.

With the limited availability of prospective data, we can glean some information from meta-analyses and pooled analyses that have been conducted in an attempt to elucidate the effects of GLP-1RAs on cardiovascular outcomes. For example, a prespecified meta-analysis, which evaluated cardiovascular risk in the dulaglutide clinical development program, ${ }^{74}$ indicated that there were no significant differences between dulaglutide and placebo groups with regard to the risk of MACE. Similarly, a meta-analysis of cardiovascular events occurring during treatment with albiglutide, placebo, 
or active comparators in the HARMONY clinical trial program $^{75}$ determined there was no significant difference between albiglutide and comparator for the risk of MACE or hospital admission for unstable angina.

The effect of exenatide BID on cardiovascular outcomes has also been assessed in a pooled meta-analysis of cardiovascular safety data from 12 long-term, randomized, placebo- or insulin comparator-controlled trials. ${ }^{76}$ The pooled relative risk for primary MACE for users of exenatide BID versus the comparator favored exenatide, suggesting no increased cardiovascular risk associated with exenatide use versus insulin or placebo. Two additional meta-analyses did not find any increase in the incidence of MACE in users of GLP-1RAs compared with comparators. ${ }^{77,78}$ Both studies showed that the GLP-1RAs were associated with a significantly lower risk of MACE compared with placebo, but not with active comparators (with the exception of pioglitazone) ${ }^{77,78}$ Consistent with these two studies, a sequential analysis of long-term trials comparing the effect of GLP-1RAs with other glucoselowering drugs or placebo ${ }^{79}$ as well as a large comparative safety study of GLP-1RAs versus other glucose-lowering agents ${ }^{80}$ both demonstrated no significant difference in the risk of cardiovascular events between drug classes.

Real-world data can also provide some insight into the impact of the GLP-1RAs on cardiovascular risk in patients with T2D. Retrospective studies have suggested that exenatide BID, with or without concomitant insulin, significantly reduced the risk of cardiovascular events compared with insulin ${ }^{81}$ and that exenatide BID is associated with a lower risk of cardiovascular events and hospitalizations versus other glucose-lowering therapies. ${ }^{82}$

The present review included prospective and retrospective studies that compared the efficacy and safety of approved GLP-1RAs with other currently available glucose-lowering therapies in the treatment of patients with T2D, with data showing clinical benefit of GLP-1RAs over other glucoselowering therapies. However, it is critical to consider the patient selection criteria, background medications, properties of the individual GLP-1RAs, drug exposure across dose intervals, and outcome definitions of each study. Furthermore, the patient characteristics in these studies need to be taken into account to assess the generalizability of the results to the T2D population at large; overly strict criteria limit the applicability of the data to everyday clinical practice.

\section{Conclusion}

This systematic analysis shows that GLP-1RAs were generally as effective as, or more effective than, oral glucose-lowering therapies in improving glycemic parameters such as $\mathrm{HbA} 1 \mathrm{c}$ and FG in patients with T2D. The reduction in $\mathrm{HbA} 1 \mathrm{c}$ with GLP-1RAs tended to be similar or smaller compared with the reductions achieved with insulin therapy, with less hypoglycemia. GLP-1RAs were consistently more effective at reducing weight than oral glucose-lowering therapies and insulin. Additionally, the GLP-1RAs appeared to have favorable effects on cardiovascular risk factors such as BP and lipid levels. In summary, GLP-1RAs are an effective, safe, and well-tolerated treatment option for T2D, with minimal risk of hypoglycemia and providing modest weight loss.

\section{Acknowledgments}

Sheridan Henness, PhD, of inScience Communications, Springer Healthcare, provided medical writing support funded by AstraZeneca. The development of this manuscript was supported by AstraZeneca.

\section{Author contributions}

All authors have contributed sufficiently to the project to be included as authors. The authors contributed equally to the conception and design of the systematic review and literature search, interpretation of the results, and drafting of the manuscript.

\section{Disclosure}

PA Levin has received grants from AstraZeneca, Boehringer Ingelheim, Eli Lilly, Merck, Novo Nordisk, and Sanofi. He is a consultant for AstraZeneca, Novo Nordisk, and Sanofi, and a speaker for AstraZeneca, Boehringer Ingelheim, Eli Lily, GlaxoSmithKline, Janssen, Novo Nordisk, and Sanofi. $\mathrm{H}$ Nguyen was an employee of AstraZeneca at the time this review was conceived and drafted. ET Wittbrodt is an employee of AstraZeneca. SC Kim has received research grants to the Brigham and Women's Hospital from AstraZeneca, Bristol-Myers Squibb, Genentech, Lilly, and Pfizer. The authors report no other conflicts of interest in this work.

\section{References}

1. American Diabetes Association. Standards of medical care in diabetes2015. Diabetes Care. 2015;38(Suppl 1):S1-S93.

2. International Diabetes Federation. IDF Diabetes Atlas, 7th Ed. 2015. Available from: http://www.diabetesatlas.org/resources/2015-atlas.html. Accessed January 31, 2017.

3. Mathers CD, Loncar D. Projections of global mortality and burden of disease from 2002 to 2030. PLoS Med. 2006;3(11):e442.

4. Inzucchi SE, Bergenstal RM, Buse JB, et al. Management of hyperglycemia in type 2 diabetes, 2015: a patient-centered approach: update to a position statement of the American Diabetes Association and the European Association for the Study of Diabetes. Diabetes Care. 2015; 38(1):140-149. 
5. Marso SP, Daniels GH, Brown-Frandsen K, et al; LEADER Steering Committee; LEADER Trial Investigators. Liraglutide and cardiovascular outcomes in type 2 diabetes. $N$ Engl J Med. 2016;375(4):311-322.

6. Pfeffer MA, Claggett B, Diaz R, et al; ELIXA Investigators. Lixisenatide in patients with type 2 diabetes and acute coronary syndrome. $N$ Engl J Med. 2015;373(23):2247-2257.

7. Berg JK, Shenouda SK, Heilmann CR, Gray AL, Holcombe JH. Effects of exenatide twice daily versus sitagliptin on 24-h glucose, glucoregulatory and hormonal measures: a randomized, double-blind, crossover study. Diabetes Obes Metab. 2011;13(11):982-989.

8. Bergenstal RM, Wysham C, Macconell L, et al; DURATION-2 Study Group. Efficacy and safety of exenatide once weekly versus sitagliptin or pioglitazone as an adjunct to metformin for treatment of type 2 diabetes (DURATION-2): a randomised trial. Lancet. 2010;376(9739):431-439.

9. Russell-Jones D, Cuddihy RM, Hanefeld M, et al; DURATION-4 Study Group. Efficacy and safety of exenatide once weekly versus metformin, pioglitazone, and sitagliptin used as monotherapy in drug-naive patients with type 2 diabetes (DURATION-4): a 26-week double-blind study. Diabetes Care. 2012;35(2):252-258.

10. Nauck M, Weinstock RS, Umpierrez GE, Guerci B, Skrivanek Z, Milicevic Z. Efficacy and safety of dulaglutide versus sitagliptin after 52 weeks in type 2 diabetes in a randomized controlled trial (AWARD-5). Diabetes Care. 2014;37(8):2149-2158.

11. Weinstock RS, Guerci B, Umpierrez G, Nauck MA, Skrivanek Z, Milicevic Z. Safety and efficacy of once-weekly dulaglutide versus sitagliptin after 2 years in metformin-treated patients with type 2 diabetes (AWARD-5): a randomized, phase III study. Diabetes Obes Metab. 2015;17(9):849-858.

12. Charbonnel B, Steinberg H, Eymard E, et al. Efficacy and safety over 26 weeks of an oral treatment strategy including sitagliptin compared with an injectable treatment strategy with liraglutide in patients with type 2 diabetes mellitus inadequately controlled on metformin: a randomised clinical trial. Diabetologia. 2013;56(7):1503-1511.

13. Pratley R, Nauck M, Bailey T, et al; 1860-LIRA-DPP-4 Study Group. One year of liraglutide treatment offers sustained and more effective glycaemic control and weight reduction compared with sitagliptin, both in combination with metformin, in patients with type 2 diabetes: a randomised, parallel-group, open-label trial. Int J Clin Pract 2011;65(4):397-407.

14. Pratley RE, Nauck M, Bailey T, et al; 1860-LIRA-DPP-4 Study Group. Liraglutide versus sitagliptin for patients with type 2 diabetes who did not have adequate glycaemic control with metformin: a 26-week, randomised, parallel-group, open-label trial. Lancet. 2010; 375(9724):1447-1456

15. Pratley RE, Nauck MA, Bailey T, et al; 1860-LIRA-DPP-4 Study Group. Efficacy and safety of switching from the DPP-4 inhibitor sitagliptin to the human GLP-1 analog liraglutide after 52 weeks in metformin-treated patients with type 2 diabetes: a randomized, open-label trial. Diabetes Care. 2012;35(10):1986-1993.

16. Takeshita Y, Takamura T, Kita Y, et al; Establishment of Rationale for Antiaging Diabetic Medicine (ERA-DM) Study Chapter 2 Group. Vildagliptin vs liraglutide as a second-line therapy switched from sitagliptin-based regimens in patients with type 2 diabetes: a randomized, parallel-group study. J Diabetes Investig. 2015;6(2):192-200.

17. Van Gaal L, Souhami E, Zhou T, Aronson R. Efficacy and safety of the glucagon-like peptide-1 receptor agonist lixisenatide versus the dipeptidyl peptidase-4 inhibitor sitagliptin in young $(<50$ years) obese patients with type 2 diabetes mellitus. $J$ Clin Transl Endocrinol. 2014;1(2):31-37.

18. Horton ES, Silberman C, Davis KL, Berria R. Weight loss, glycemic control, and changes in cardiovascular biomarkers in patients with type 2 diabetes receiving incretin therapies or insulin in a large cohort database. Diabetes Care. 2010;33(8):1759-1765.

19. Montilla S, Marchesini G, Sammarco A, et al; AIFA Anti-diabetics Monitoring Group. Drug utilization, safety, and effectiveness of exenatide, sitagliptin, and vildagliptin for type 2 diabetes in the real world: data from the Italian AIFA Anti-diabetics Monitoring Registry. Nutr Metab Cardiovasc Dis. 2014;24(12):1346-1353.
20. Nyeland ME, Ploug UJ, Richards A, et al. Evaluation of the effectiveness of liraglutide and sitagliptin in type 2 diabetes: a retrospective study in UK primary care. Int J Clin Pract. 2015;69(3):281-291.

21. Umpierrez G, Tofé Povedano S, Pérez Manghi F, Shurzinske L, Pechtner $\mathrm{V}$. Efficacy and safety of dulaglutide monotherapy versus metformin in type 2 diabetes in a randomized controlled trial (AWARD-3). Diabetes Care. 2014;37(8):2168-2176.

22. Derosa G, Maffioli P, Salvadeo SA, et al. Exenatide versus glibenclamide in patients with diabetes. Diabetes Technol Ther. 2010;12(3):233-240.

23. Derosa G, Putignano P, Bossi AC, et al. Exenatide or glimepiride added to metformin on metabolic control and on insulin resistance in type 2 diabetic patients. Eur J Pharmacol. 2011;666(1-3):251-256.

24. Gallwitz B, Guzman J, Dotta F, et al. Exenatide twice daily versus glimepiride for prevention of glycaemic deterioration in patients with type 2 diabetes with metformin failure (EUREXA): an open-label, randomised controlled trial. Lancet. 2012;379(9833):2270-2278.

25. Garber A, Henry R, Ratner R, et al; LEAD-3 (Mono) Study Group. Liraglutide versus glimepiride monotherapy for type 2 diabetes (LEAD-3 Mono): a randomised, 52-week, phase III, double-blind, parallel-treatment trial. Lancet. 2009;373(9662):473-481.

26. Garber A, Henry RR, Ratner R, Hale P, Chang CT, Bode B; LEAD-3 (Mono) Study Group. Liraglutide, a once-daily human glucagon-like peptide 1 analogue, provides sustained improvements in glycaemic control and weight for 2 years as monotherapy compared with glimepiride in patients with type 2 diabetes. Diabetes Obes Metab. 2011; 13(4):348-356.

27. Kaku K, Rasmussen MF, Nishida T, Seino Y. Fifty-two-week, randomized, multicenter trial to compare the safety and efficacy of the novel glucagon-like peptide-1 analog liraglutide vs glibenclamide in patients with type 2 diabetes. J Diabetes Investig. 2011;2(6):441-447.

28. Nauck M, Frid A, Hermansen K, et al. Long-term efficacy and safety comparison of liraglutide, glimepiride and placebo, all in combination with metformin in type 2 diabetes: 2-year results from the LEAD-2 study. Diabetes Obes Metab. 2013;15(3):204-212.

29. Nauck M, Marre M. Adding liraglutide to oral antidiabetic drug monotherapy: efficacy and weight benefits. Postgrad Med. 2009;121(3):5-15.

30. Seino Y, Rasmussen MF, Nishida T, Kaku K. Efficacy and safety of the once-daily human GLP-1 analogue, liraglutide, vs glibenclamide monotherapy in Japanese patients with type 2 diabetes. Curr Med Res Opin. 2010;26(5):1013-1022.

31. Chiefari E, Capula C, Vero A, et al. Add-on treatment with liraglutide improves glycemic control in patients with type 2 diabetes on metformin therapy. Diabetes Technol Ther. 2015;17(7):468-474.

32. Nauck M, Frid A, Hermansen K, et al; LEAD-2 Study Group. Efficacy and safety comparison of liraglutide, glimepiride, and placebo, all in combination with metformin, in type 2 diabetes: the LEAD (liraglutide effect and action in diabetes)-2 study. Diabetes Care. 2009;32(1): 84-90.

33. Marre M, Shaw J, Brändle M, et al; LEAD-1 SU study group. Liraglutide, a once-daily human GLP-1 analogue, added to a sulphonylurea over 26 weeks produces greater improvements in glycaemic and weight control compared with adding rosiglitazone or placebo in subjects with type 2 diabetes (LEAD-1 SU). Diabet Med. 2009;26(3):268-278.

34. DeFronzo RA, Triplitt C, Qu Y, Lewis MS, Maggs D, Glass LC. Effects of exenatide plus rosiglitazone on beta-cell function and insulin sensitivity in subjects with type 2 diabetes on metformin. Diabetes Care. 2010;33(5):951-957.

35. Xu W, Bi Y, Sun Z, et al. Comparison of the effects on glycaemic control and $\beta$-cell function in newly diagnosed type 2 diabetes patients of treatment with exenatide, insulin or pioglitazone: a multicentre randomized parallel-group trial (the CONFIDENCE study). J Intern Med. 2015;277(1):137-150.

36. Araki E, Inagaki N, Tanizawa Y, Oura T, Takeuchi M, Imaoka T. Efficacy and safety of once-weekly dulaglutide in combination with sulphonylurea and/or biguanide compared with once-daily insulin glargine in Japanese patients with type 2 diabetes: a randomized, open-label, phase III, non-inferiority study. Diabetes Obes Metab. 2015;17(10): 994-1002. 
37. Barnett AH, Burger J, Johns D, et al. Tolerability and efficacy of exenatide and titrated insulin glargine in adult patients with type 2 diabetes previously uncontrolled with metformin or a sulfonylurea: a multinational, randomized, open-label, two-period, crossover noninferiority trial. Clin Ther. 2007;29(11):2333-2348.

38. Bergenstal R, Lewin A, Bailey T, Chang D, Gylvin T, Roberts V; NovoLog Mix-vs.-Exenatide Study Group. Efficacy and safety of biphasic insulin aspart 70/30 versus exenatide in subjects with type 2 diabetes failing to achieve glycemic control with metformin and a sulfonylurea. Curr Med Res Opin. 2009;25(1):65-75.

39. Blonde L, Jendle J, Gross J, et al. Once-weekly dulaglutide versus bedtime insulin glargine, both in combination with prandial insulin lispro, in patients with type 2 diabetes (AWARD-4): a randomised, open-label, phase 3, non-inferiority study. Lancet. 2015;385(9982):2057-2066.

40. D'Alessio D, Häring HU, Charbonnel B, et al; EAGLE Investigators. Comparison of insulin glargine and liraglutide added to oral agents in patients with poorly controlled type 2 diabetes. Diabetes Obes Metab. 2015;17(2):170-178.

41. Davies M, Heller S, Sreenan S, et al. Once-weekly exenatide versus once- or twice-daily insulin detemir: randomized, open-label, clinical trial of efficacy and safety in patients with type 2 diabetes treated with metformin alone or in combination with sulfonylureas. Diabetes Care. 2013;36(5):1368-1376.

42. Davies MJ, Donnelly R, Barnett AH, Jones S, Nicolay C, Kilcoyne A. Exenatide compared with long-acting insulin to achieve glycaemic control with minimal weight gain in patients with type 2 diabetes: results of the Helping Evaluate Exenatide in patients with diabetes compared with Long-Acting insulin (HEELA) study. Diabetes Obes Metab. 2009;11(12):1153-1162.

43. Diamant M, Nauck MA, Shaginian R, et al; 4B Study Group. Glucagonlike peptide 1 receptor agonist or bolus insulin with optimized basal insulin in type 2 diabetes. Diabetes Care. 2014;37(10):2763-2773.

44. Diamant M, Van Gaal L, Guerci B, et al. Exenatide once weekly versus insulin glargine for type 2 diabetes (DURATION-3): 3-year results of an open-label randomised trial. Lancet Diabetes Endocrinol. 2014; 2(6):464-473.

45. Diamant M, Van Gaal L, Stranks S, et al. Safety and efficacy of onceweekly exenatide compared with insulin glargine titrated to target in patients with type 2 diabetes over 84 weeks. Diabetes Care. 2012; 35(4):683-689.

46. Diamant M, Van Gaal L, Stranks S, et al. Once weekly exenatide compared with insulin glargine titrated to target in patients with type 2 diabetes (DURATION-3): an open-label randomised trial. Lancet. 2010;375(9733):2234-2243.

47. Gallwitz B, Böhmer M, Segiet T, et al. Exenatide twice daily versus premixed insulin aspart 70/30 in metformin-treated patients with type 2 diabetes: a randomized 26-week study on glycemic control and hypoglycemia. Diabetes Care. 2011;34(3):604-606.

48. Giorgino F, Benroubi M, Sun JH, Zimmermann AG, Pechtner V. Efficacy and safety of once-weekly dulaglutide versus insulin glargine in patients with type 2 diabetes on metformin and glimepiride (AWARD-2). Diabetes Care. 2015;38(12):2241-2249.

49. Gough SC, Bode BW, Woo VC, et al. One-year efficacy and safety of a fixed combination of insulin degludec and liraglutide in patients with type 2 diabetes: results of a 26-week extension to a 26-week main trial. Diabetes Obes Metab. 2015;17(10):965-973.

50. Gough SCL, Bode B, Woo V, et al; NN9068-3697 (DUAL-I) trial investigators. Efficacy and safety of a fixed-ratio combination of insulin degludec and liraglutide (IDegLira) compared with its components given alone: results of a phase 3, open-label, randomised, 26-week, treat-to-target trial in insulin-naive patients with type 2 diabetes. Lancet Diabetes Endocrinol. 2014;2(11):885-893.

51. Heine RJ, Van Gaal LF, Johns D, Mihm MJ, Widel MH, Brodows RG; GWAA Study Group. Exenatide versus insulin glargine in patients with suboptimally controlled type 2 diabetes: a randomized trial. Ann Intern Med. 2005;143(8):559-569.
52. Inagaki N, Atsumi Y, Oura T, Saito H, Imaoka T. Efficacy and safety profile of exenatide once weekly compared with insulin once daily in Japanese patients with type 2 diabetes treated with oral antidiabetes drug(s): results from a 26-week, randomized, open-label, parallel-group, multicenter, noninferiority study. Clin Ther. 2012;34(9): 1892-1908.

53. Mathieu C, Ostenson CG, Matthaei S, et al. Using exenatide twice daily or insulin in clinical practice: results from CHOICE. Diabetes Ther. 2013;4(2):285-308.

54. Mathieu C, Rodbard HW, Cariou B, et al; BEGIN: VICTOZA ADD-ON (NN1250-3948) study group. A comparison of adding liraglutide versus a single daily dose of insulin aspart to insulin degludec in subjects with type 2 diabetes (BEGIN: VICTOZA ADD-ON). Diabetes Obes Metab. 2014;16(7):636-644.

55. Nauck MA, Duran S, Kim D, et al. A comparison of twice-daily exenatide and biphasic insulin aspart in patients with type 2 diabetes who were suboptimally controlled with sulfonylurea and metformin: a non-inferiority study. Diabetologia. 2007;50(2):259-267.

56. Ostenson CG, Matthaei S, Reaney M, et al. Treatment outcomes after initiation of exenatide twice daily or insulin in clinical practice: 12-month results from CHOICE in six European countries. Diabetes Metab Syndr Obes. 2013;6:171-185.

57. Russell-Jones D, Vaag A, Schmitz O, et al; Liraglutide Effect and Action in Diabetes 5 (LEAD-5) met+SU Study Group. Liraglutide vs insulin glargine and placebo in combination with metformin and sulfonylurea therapy in type 2 diabetes mellitus (LEAD- 5 met+SU): a randomised controlled trial. Diabetologia. 2009;52(10):2046-2055.

58. Baser O, Wei W, Baser E, Xie L. Clinical and economic outcomes in patients with type 2 diabetes initiating insulin glargine disposable pen versus exenatide BID. J Med Econ. 2011;14(6):673-680.

59. Bounthavong M, Tran JN, Golshan S, et al. Retrospective cohort study evaluating exenatide twice daily and long-acting insulin analogs in a Veterans Health Administration population with type 2 diabetes. Diabetes Metab. 2014;40(4):284-291.

60. Dalal MR, Xie L, Baser O, DiGenio A. Adding rapid-acting insulin or GLP-1 receptor agonist to basal insulin: outcomes in a community setting. Endocr Pract. 2015;21(1):68-76.

61. Pawaskar M, Li Q, Hoogwerf BJ, et al. Metabolic outcomes of matched patient populations initiating exenatide BID vs. insulin glargine in an ambulatory care setting. Diabetes Obes Metab. 2012;14(7): 626-633.

62. Pawaskar M, Li Q, Reynolds MW. Metabolic outcomes of elderly patient populations initiating exenatide BID versus insulin glargine in an ambulatory care setting. Curr Med Res Opin. 2012;28(6): 991-997.

63. Sudhakaran C, Fathima M, Anjana RM, Unnikrishnan RI, Mohan V. Effectiveness of exenatide in Asian Indians in a clinical care setting. Diabetes Technol Ther. 2010;12(8):613-618.

64. Reid T. Choosing GLP-1 receptor agonists or DPP-4 inhibitors: weighing the clinical trial evidence. Clin Diabetes. 2012;30(1):3-12.

65. Kim W, Egan JM. The role of incretins in glucose homeostasis and diabetes treatment. Pharmacol Rev. 2008;60(4):470-512.

66. Smilowitz NR, Donnino R, Schwartzbard A. Glucagon-like peptide-1 receptor agonists for diabetes mellitus: a role in cardiovascular disease. Circulation. 2014;129(22):2305-2312.

67. Lang K, Nguyen H, Huang H, Kaufman E, Levin P. Real-world treatment responses from electronic medical record (EMR) data among patients with type 2 diabetes (T2D) receiving basal insulin either with mealtime insulin or exenatide BID, by A1c attainment level and baseline A1c [abstract 1540-P]. Diabetes. 2016;65(Suppl 1):A360-A431.

68. Lorenz M, Lawson F, Owens D, et al. Differential effects of glucagonlike peptide-1 receptor agonists on heart rate. Cardiovasc Diabetol. 2017;16(1):6.

69. Marso SP, Bain SC, Consoli A, et al; SUSTAIN-6 Investigators. Semaglutide and cardiovascular outcomes in patients with type 2 diabetes. N Engl J Med. 2016;375(19):1834-1844. 
70. Bentley-Lewis R, Aguilar D, Riddle MC, et al; ELIXA Investigators. Rationale, design, and baseline characteristics in Evaluation of LIXisenatide in Acute Coronary Syndrome, a long-term cardiovascular end point trial of lixisenatide versus placebo. Am Heart J. 2015; 169(5):631-638.

71. Holman RR, Bethel MA, George J, et al. Rationale and design of the EXenatide Study of Cardiovascular Event Lowering (EXSCEL) trial. Am Heart J. 2016;174:103-110.

72. Eli Lilly and Company. The effect of dulaglutide on major cardiovascular events in patients with type 2 diabetes: Researching Cardiovascular Events with a Weekly INcretin in Diabetes (REWIND). ClinicalTrials. gov record: NCT01394952. Available from: https://clinicaltrials.gov/ ct2/show/NCT01394952. Accessed October 11, 2016.

73. GlaxoSmithKline. A long term, randomised, double blind, placebocontrolled study to determine the effect of albiglutide, when added to standard blood glucose lowering therapies, on major cardiovascular events in patients with type 2 diabetes mellitus (HARMONY Outcomes). ClinicalTrials.gov identifier: NCT02465515. Available from: https://clinicaltrials.gov/ct2/show/NCT02465515. Accessed October 11, 2016.

74. Ferdinand KC, Botros FT, Atisso CM, Sager PT. Cardiovascular safety for once-weekly dulaglutide in type 2 diabetes: a pre-specified metaanalysis of prospectively adjudicated cardiovascular events. Cardiovasc Diabetol. 2016;15:38.

75. Fisher M, Petrie MC, Ambery PD, Donaldson J, Ye J, McMurray JJ. Cardiovascular safety of albiglutide in the Harmony programme: a meta-analysis. Lancet Diabetes Endocrinol. 2015;3(9):697-703.
76. Ratner R, Han J, Nicewarner D, Yushmanova I, Hoogwerf BJ, Shen L. Cardiovascular safety of exenatide BID: an integrated analysis from controlled clinical trials in participants with type 2 diabetes. Cardiovasc Diabetol. 2011;10:22.

77. Monami M, Cremasco F, Lamanna C, et al. Glucagon-like peptide-1 receptor agonists and cardiovascular events: a meta-analysis of randomized clinical trials. Exp Diabetes Res. 2011;2011:215764.

78. Monami M, Dicembrini I, Nardini C, Fiordelli I, Mannucci E. Effects of glucagon-like peptide-1 receptor agonists on cardiovascular risk: a meta-analysis of randomized clinical trials. Diabetes Obes Metab. 2014; 16(1):38-47.

79. Wu S, Sun F, Zhang Y, et al. The cardiovascular effects of glucagon-like peptide-1 receptor agonists: a trial sequential analysis of randomized controlled trials. J Clin Pharm Ther. 2014;39(1):7-13.

80. Patorno E, Everett BM, Goldfine AB, et al. Comparative cardiovascular safety of glucagon-like peptide-1 receptor agonists versus other antidiabetic drugs in routine care: a cohort study. Diabetes Obes Metab. 2016;18(8):755-765.

81. Paul SK, Klein K, Maggs D, Best JH. The association of the treatment with glucagon-like peptide-1 receptor agonist exenatide or insulin with cardiovascular outcomes in patients with type 2 diabetes: a retrospective observational study. Cardiovasc Diabetol. 2015;14:10.

82. Best JH, Hoogwerf BJ, Herman WH, et al. Risk of cardiovascular disease events in patients with type 2 diabetes prescribed the glucagonlike peptide 1 (GLP-1) receptor agonist exenatide twice daily or other glucose-lowering therapies: a retrospective analysis of the LifeLink database. Diabetes Care. 2011;34(1):90-95.
Diabetes, Metabolic Syndrome and Obesity: Targets and Therapy is an international, peer-reviewed open-access journal committed to the rapid publication of the latest laboratory and clinical findings in the fields of diabetes, metabolic syndrome and obesity research Original research, review, case reports, hypothesis formation, expert

\section{Dovepress}

opinion and commentaries are all considered for publication. The manuscript management system is completely online and includes a very quick and fair peer-review system, which is all easy to use. Visit http://www.dovepress.com/testimonials.php to read real quotes from published authors. 